\title{
LES SOCIÉTÉS MATRISTIQUES CHINOISES: UN CAS DE PLURALISME JURIDIQUE
}

CHINESE MATRISTIC SOCIETIES: A CASE OF LEGAL PLURALISM

NORBERT ROULAND"

\section{RÉSUMÉ}

Les $\mathrm{Na}$ sont une minorité nationale du Sud de la Chine d'environ 30000 individus. Leurs coutumes matrimoniales sont un cas de pluralisme juridique. Elles sont matrilinéaires, ce qu'il ne faut pas confondre avec un système matriarcal qui n'a jamais existé. L'ethnie dominante chinoise, les Han, sont au contraire patriarcaux. Ils n'ont jamais compris le système $\mathrm{Na}$, notamment les visites furtives , qu'ils ont assimilées à de la prostitution. Un tragique malentendu culturel sur la notion de famille. Le gouvernement central chinois , notamment pendant la Révolution culturelle, a plusieurs fois essayé de détruire les coutumes des $\mathrm{Na}$, sans jamais y parvenir totalement.

MOTS-CLEFS: Matrilinéarité. Matriarcat. Patriarcat. Révolution Culturelle. Visites furtives.

\begin{abstract}
The $\mathrm{Na}$ is a national minority in southern China of around 30,000 people. Their matrimonial customs are a case of legal pluralism. They are matrilineal, which should not be confused with a matriarchal system that never existed. On the contrary, the dominant Chinese ethnicity, the Han, are patriarchal. They never understood the $\mathrm{Na}$ system, especially the stealthy visits, which they equated to prostitution. A tragic cultural misunderstanding about the concept of family. The Chinese central government, especially during the Cultural Revolution, has tried several times to destroy the customs of the $\mathrm{Na}$, but never fully succeeded.
\end{abstract}

KEYWORDS: Matrilineality, Matriarchy, Patriarchy, Cultural Revolution, Stealth visits

L'un des plus grands et peut-être le principal fondement des Républiques est d'accommoder l'estat au naturel des citoyens, et les édits et les ordonnances à la nature des lieux, des personnes et du temps (...) qui fait aussi qu'on doit diversifier l'estat de la République à la diversité des lieux, à l'exemple du bon architecte qui accommode son bâtiment à la matière qu'il trouve sur les lieux.

Jean Bodin, La République, V-1.

Il y a de certaines idées d'uniformité qui saisissent quelquefois les grands esprits (...) mais qui frappent infailliblement les petits (...) La grandeur du génie ne consisterait-elle pas à savoir dans quels cas il faut l'uniformité, et dans quels cas il faut des différences? (...) Lorsque les citoyens suivent les lois, qu'importe qu'ils suivent la même?

Montesquieu, L'Esprit des lois, XXXIX , 18.

* Professeur émérite à la Faculté de droit d'Aix en Provence, Ancien Membre de l'Institut Universitaire de France, Chaire d'Anthropologie Juridique.

E-mail: : norbert.rouland@wanadoo.fr 
Le législateur ne doit point perdre de vue que les lois sont faites pour les hommes, et non les hommes pour les lois; qu'elles doivent être adaptées au caractère, aux habitudes, à la situation du peuple pour lequel elles sont faites.

Portalis, Discours préliminaire prononcé lors de la présentation $d u$ projet de Code civil, An XI.

\section{INTRODUCTION}

Le pluralisme juridique est un sujet qui a fait couler beaucoup d'encre, dans des écrits scientifiques peu lus en France, car la France, qui a été le premier des Etats européens à être centralisé, est demeuré un État unitaire qui refuse de reconnaître l'existence de minorités ou de peuples autochtones, au moins sur le sol métropolitain. Ce qui ne l'empêche pas d'admettre une certaine diversité des statuts: l'article 75 de la Constitution permet à certaines populations d'outremer de conserver leur statut personnel ${ }^{1}$. Pour des raisons évidemment différentes, il en va de même de la notion de matriarcat, qui demeure aujourd'hui encore obscurcie par un certain nombre d'erreurs et de préjugés. Dans la première partie de cette communication, je tenterai donc de clarifier ces deux concepts. Mais l'essentiel de cette communication sera consacré à des aspects beaucoup moins théoriques, et reposera dans une seconde partie sur les résultats d'enquêtes de terrain menées dans une société dite matriarcale (mieux vaudrait parler de société matristique) de la Chine du Sud, où je dois me rendre en 2023. Il s'agit des $\mathrm{Na}$, auquel est consacré un ouvrage qui a connu une grande notoriété, écrit par le Pr. Cai Hua, un collègue chinois, à la fin du siècle précédent, : Les Na,une société son père, ni mari. Une exception anthropologique ? En tout cas, cette société a suscité l'étonnement de voyageurs occidentaux, mais aussi de nombreux gouvernement chinois au cours de l'histoire. Qu'en a t'-il été au cours des dernières décennies ? Il faudra s'interroger sur les politiques du gouvernement chinois qui a tenté, sans succès, d'abolir ce droit coutumier. Je terminerai par quelques réflexions montrant que la politique chinoise n'a rien d'exceptionnel.

1 Cf. Norbert ROULAND, Les statuts personnels et les droits coutumiers dans le droit constitutionnel français, dans : Anne-Marie LE POURHIET(dir.),Préface de Louis Favoreu, Droit constitutionnel local, Economica, Puam, Paris et Aix en Provence, 1999, 145-226. Avec un commentaire de Didier MAUSS. 


\section{PARTIE I : LES CONCEPTS}

Je parlerai d'abord du pluralisme juridique, ensuite du matriarcat.

\section{A) QUID DU PLURALISME JURIDIQUE ?}

Le pluralisme juridique a donné lieu à beaucoup de variations théoriques. A elles seules, elles pourraient faire l'objet de plusieurs articles. Je me bornerai donc ici à l'essentiel'2. Il s'agit d'un ensemble de doctrines mettant en cause l'identification exclusive du droit à l'État. Les auteurs les plus représentatifs sont Kelsen et Carré de Malberg. La doctrine française en est un bon exemple. Mais d'autres auteurs, à partir de raisonnements divers, pensent que le droit peut exister en dehors de l'État et de la sanction par ses tribunaux. En France, pour Duguit et Hauriou, la vie sociale est organisée par une multitude de groupements, ou institutions, qui produisent leurs propres droits. On trouve des théories analogues chez les anthropologues du droit comme Léopold Pospisil, Jean-Guy Belley au Canada, John Griffiths, Jacques Vanderlinden, Masaji Chiba au Japon.

Je voudrais signaler deux erreurs fréquemment commises au sujet du pluralisme juridique. La première est la confusion entre pluralisme juridique et diversité juridique. Même les systèmes les plus unitaires, comme le droit français, ou le droit chinois, admettent une certaine dose de diversité. Il existe en droit français des discriminations positives et en Nouvelle-Calédonie est en train de se former un droit civil coutumier $\mathrm{kanak}^{3}$. Mais cette formation est une variation autorisée par le droit de l'État. Le pluralisme juridique va beaucoup plus loin, puisqu'il part de l'idée de la coexistence plus ou moins pacifique entre différents systèmes juridiques, parmi lesquels le droit étatique.

La seconde erreur consiste à postuler la primauté du droit étatique sur tous les autres systèmes juridiques. Tout dépend de la situation de l'observateur. Il en va bien ainsi pour les représentants du droit étatique : juges, policiers, administrateurs, etc. Mais il n'en est pas de même pour les individus qui appartiennent aux groupements précédemment indiqués. Pour eux, c'est leur propre droit qui prime, et ils l'appliquent, comme nous le verrons dans le cas des $\mathrm{Na}$, du sud de la Chine.

C'est sur ce point que s'opère la césure entre les auteurs favorables au pluralisme juridique et le doyen Jean Carbonnier, un auteur pourtant très ouvert à la sociologie et à l'anthropologie juridiques. Il dénonce : «la grande illusion $d u$ pluralisme. Il croit avoir filmé le combat de deux systèmes juridiques ; mais ce

2 Cf. Hugues MOUTOUH, Pluralisme juridique, dans :Denis ALLAND et Stéphane RIALS(dir ;), Dictionnaire de la culture juridique, Paris, Presses universitaires de France, 2003,11 58-11 62.

3 Cf. Antoine LECA, Précis de droit civil coutumier kanak, Centre de documentation pédagogique de Nouvelle Calédonie, Nouméa, 2018. Cf. aussi :Léon WAMYTAN, Peuple Kanak et droit français, Centre de documentation pédagogique de Nouvelle Calédonie, Nouméa, 2013. 
qu'il montre est un système juridique aux prises avec l'ombre d'un autre ». Il ne suffit pas que les individus croient qu'ils agissent suivant des normes juridiques pour qu'elles le soient. Elles ne le sont que lorsqu'elles sont sanctionnables par des tribunaux. Le doyen Carbonnier, en ce sens, reste donc fidèle à la doctrine française. On peut lui opposer d'une part que sa position privilégie un point de vue externe aux manifestations de normes de comportements internes, ou des institutions, au sens d'Hauriou. D'autre part, la sanction n'est pas le seul critère de l'existence du droit. Des anthropologues comme Malinowski ont montré que l'homme peut vivre le droit en dehors du conflit : ce sont les rapports réciproques d'obligations, peut-être davantage que la contrainte, qui assurent la cohérence de la société.

En réalité, comme il n'existe pas de preuves empiriques de la nature et de l'existence du droit, le pluralisme comme le monisme juridiques sont indécidables. La position de l'observateur théoricien modifie celle du concept observé. Les théories ne tombent pas du ciel comme le voudrait l'idéalisme juridique, mais elles s'inscrivent dans des lieux et des moments de l'histoire, sont utilisées par des acteurs, du monde politique à celui des universités.

L'exemple de l'un des principaux théoriciens européens du pluralisme juridique, Cornelis Van Vollenhoven (1874-19 33), le montre bien ${ }^{4}$. Il est le fondateur de l'école hollandaise du droit coutumier, Adat Law School. Professeur de droit, il fut aussi le secrétaire de J.Th. Cremer, Ministre des affaires coloniales. On peut le considérer comme le fondateur du pluralisme juridique. Il refuse de considérer la sanction comme le critère du droit. En 1901, au sujet de l'Indonésie, colonie hollandaise jusqu'à la fin du second conflit mondial, il considère que les villages, les clans, produisent eux-mêmes leurs droits, qui sont opposables au droit colonial. A l'époque, c'était révolutionnaire. Il intervient plusieurs fois auprès de l'administration, se posant en défenseur des populations indonésiennes, défendant notamment les droits des autochtones sur leurs terres. En 1937, il anticipe de vint ans la controverse entre Gluckman et Bohannan(voir la thése en chinois sur ce sujet du Pr. Wang Weichen, de Shanghai), célèbre en anthropologie juridique, en insistant sur le fait que l'anthropologue du droit doit utiliser les catégories juridiques de la pensée et de la langue autochtones.

On voit donc que le pluralisme juridique n'est pas un concept aseptique et irénique. Il s'inscrit dans une dynamique qu'il faut bien qualifier la plupart du temps de politique, sous peine de ne rien y comprendre. Historiquement, il a donc servi d'outil dans la contestation de certaines modalités de la colonisation. On pourrait aussi dire qu'il s'inscrit dans une conception polyarchique du droit qui est évidemment à l'opposé des archétypes des Etats unitaires.

4 Cf. Norbert ROULAND, Anthropologie juridique, Paris, Presses universitaires de France, 19 88,109-111. 
Enfin, il correspond aussi à des stratégies universitaires. Reconnaître l'existence du pluralisme juridique, c'est aussi légitimer l'existence des anthropologues du droit. Ce n'est pas un hasard si leur principale association internationale a choisi de se nommer Commission on folk Law and legal pluralism.

\section{B) QUID DU MATRIARCAT ?5}

Afin d'éviter des confusions, deux observations préliminaires.

Tout d'abord, le matriarcat n'est pas le symétrique inverse du patriarcat. L'observation empirique montre que ce que nous appelons des sociétés matriarcales sont en fait des sociétés où femmes et hommes sont sur un pied d'égalité. Si le matriarcat devait être une domination des femmes sur les hommes, il n'existerait effectivement pas.Le terme de matriarcat suscite donc des confusions, et il faudrait en trouver un autre.

D'autre part, la matrilinéarité n'est pas synonyme de matriarcat. Elle signifie seulement qu'en matière d'exercice de l'autorité parentale et de transmission des biens, ce sont les lignages maternels qui sont privilégiés. C'est ainsi qu'au sein d'une famille le rôle éducatif du mari de la mère, soit le père biologique de l'enfant, est exercé par l'oncle maternel. Mais la matrilinéarité peut très bien aller de pair avec le patriarcat : au sein des lignées maternelles, ce sont les hommes qui exercent le pouvoir. Il reste que la plupart du temps les systèmes matriarcaux sont aussi des systèmes matrilinéaires.

En effet, les sociétés traditionnelles se conçoivent comme un ensemble de groupes divisés, mais complémentaires, d'où leur préférence pour l'organisation lignagère. À chaque génération sortent du lignage les descendants par les hommes ou les descendants par les femmes, et l'unilinéarité assure la prédominance d'un type de filiation sur l'autre. Sous l'influence de diverses circonstances, comme la colonisation, ou la politique d'une ethnie dominante, les deux lignes, paternelle et maternelle, vont acquérir une importance comparable. Au lieu d'être relié prioritairement à ses ascendants paternels et maternels, l'enfant est rattaché de façon égale à ses père et mère, même si dans la pratique, on privilégie dans la mémoire familiale le lignage le plus valorisé socialement ${ }^{6}$. Car il ya a une interprétation subjective des liens de parenté. Comme l'écrit C. Lévi-Strauss :

«Un système de parenté ne consiste donc pas dans des liens objectifs de filiation et de consanguinité (...) il n'existe que dans la conscience des hommes, il est un système arbitraire de représentations» ${ }^{7}$.

5 Pour un bon résumé, cf. Maryléne PATOU-MATHIS,Sortir la femme préhistorique de l'ombre, Le Monde Diplomatique, Octobre 2020,18. Elle cite notamment les travaux du Pr CAI HUA sur les $\mathrm{Na}$.

6 Cf. N.ROULAND, Palimpsestes familiaux, à paraître dans la Revue de la recherche Juridique, Aix en Provence, 2021-1.

7 Claude LEVI-STRAUSS, Anthropologie structurale, Paris, 1958,61. 
Au sein de nos généalogies, nous classons donc nos parents suivant leur statut. Nous ne nous souvenons davantage de ceux qui ont joué un rôle dans l'histoire, fût-elle locale, ou qui ont occupé une position sociale élevée. Cette équivalence des lignages et leur concentration dans la famille nucléaire sont les phases préalables à leur dissolution. Au terme de cette acculturation, la division entre les groupes anciens a disparu 8 .

\section{a) Définitions}

Que faut-il entendre, à l'heure actuelle, par société matriarcale?"

L'archéologue lituanienne Marija Gimbutas (1921-1994) ${ }^{10}$, pour éviter les confusions, préfère employer le terme de sociétés matristiques. Ces sociétés ont duré jusqu'en 3000 avant notre ère, époque à laquelle le patriarcat s'instaure avec l'invention de l'agriculture.

Au début du néolithique, l'organisation socio- économique des premières sociétés agricoles semble s'élaborer avec les femmes ${ }^{11}$. Agricultrices, elles seraient à l'origine de la domestication des plantes et de l'invention d'outils agricoles. Aux alentours de 6000 avant notre ère, la sédentarisation augmente, ainsi que la production de nourriture. Les hommes deviennent progressivement détenteurs des récoltes et des troupeaux et instaurent la filiation patrilinéaire afin d'en assurer la transmission à leurs enfants. Les femmes se retrouvent de plus en plus cantonnées dans l'espace domestique. Mais les lourds travaux ne leur sont pas épargnés. On a analysé leur état de santé à partir de squelettes féminins mis à jour à l'heure actuelle. On note une augmentation des pathologies liées au port de lourdes charges, à des grossesses répétées, à des carences dûes à une alimentation déficiente en protéines, à base principalement de féculents et de végétaux, ainsi que des traumatismes consécutifs à des violences.Le patriarcat est né. Ceci dit, le phénomène n'est pas uniforme. Dans plusieurs tombes, les défuntes portent de riches ornements et présentent peu de pathologies et de traumatismes ${ }^{12}$.

Dans un ouvrage recent,H.Goettner-Abendroth ${ }^{13}$ définit ainsi ce qu'elle nomme sociétés matriarcales :

8 Cf. N.ROULAND, op.cit.(AnthropologieJuridique), 369-370.

9 Cf. Heide GOETTNER-ABENDROTH, Les sociétés matriarcales-Recherches sur les cultures autochtones à travers le monde, Paris, Éditions des femmes, 2019,102-104, 143-144,204,220221,237,347-348,382-383, 454, 528-529.

10 Cf. Marina GIMBUTAS, Bronze Age Cultures of Central and Eastern Europe, Mouton and Co., Paris, 1965.

11 Cf. Jacques CAUVIN, Naissance des divinités, naissance de l'agriculture, :la révolution des symboles au Néolithique, Flammarion, Paris, 1998.

12 Cf. Anne AUGEREAU, La condition des femmes au Néolithique. Pour une approche du genre dans le Néolithique européen. Soutenance HDR, Institut national d'Histoire de l'art, Paris, 28 Janvier 2019.

13 Cf. Heide GOETTNER-ABENDROTH, op.cit., 20-21. 
"Les conditions nécessaires concernant la définition du matriarcat sont les suivantes : les mères sont au centre de la société, comme en atteste la matrilinéarité et, en économie, le pouvoir de distribution des biens détenus par les mères (ou les femmes) qui se situent l'une et l'autre dans un contexte d'égalité de genre. (...) La matrilinéarité est est un élément essentiel, non seulement parce qu'elle structure les relations sociales de la société tout entière, mais aussi parce qu'elle garantit ces relations via le droit d'héritage en ligne féminine et les processus de prises de décision politiques qui en découlent (...) l'égalité de genre est également un élément essentiel, car elle garantit que, nonobstant la position centrale des femmes, les sociétés matriarcales n'adoptent pas une hiérarchie de genre: elles accordent une égale valeur à l'un et l'autre sexe. Les sociétés matriarcales ne sont donc pas une image inversée du patriarcat. Cependant, matrilinéarité et égalité entre les sexes ne sont encore pas suffisantes pour caractériser le matriarcat : il faut en outre que le pouvoir de distribution économique soit détenu par les femmes. Cet élément est essentiel, parce qu'il est au fondement de l'économie matriarcale de partage. Ce type d'économie ne pourrait survenir si l'économie reposait entre les mains d'hommes et de chefs qui accumulent les biens, même si la société était matrilinéaire par ailleurs. De telles sociétés existent bien, mais elles sont seulement matrilinéaires, pas matriarcales.”

Depuis les ouvrages de Bachofen et Morgan de la fin du XIX siécle, les ethnologues ont recensé dans l'ensemble du monde environ dix mille sociétés humaines, passées ou présentes. Dans aucune les femmes n’ont le pouvoir politique, ou même un pouvoir comparable à celui des hommes. Avec le développement de sociétés sédentaires de plus en plus nombreuses, se sont multipliées les idéologies patriarcales. Le tournant paraît se situer à partir du cinquième millénaire. Les représentations féminines passent à l'arrière-plan et celles de guerriers en armes, du pouvoir masculin, deviennent le thème privilégié des images publiques.

En ce sens, si l'on suit les théories de certains auteurs actuels, le passage à l'agriculture et à la sédentarisation serait non pas un progrès, mais une régression catastrophique. De là dateraient l'accroissement de la durée et de la pénibilité du travail, des épidémies,de la montée de sociétés guerrières. En ce sens, la disparition des premières sociétés matriarcales coïnciderait avec ce tournant fatal pris par l'humanité14, même si l'histoire montre que ces premiers Etats ont souvent été fragiles et se sont effondrés au bout d'un certain temps : l'histoire n'est pas linéaire.

Sur le plan économique, les matriarcats sont généralement, mais pas toujours, des sociétés agricoles. Les champs et les maisons sont des biens communs au clan. Les droits d'usufruit l'emportent sur le droit de propriété privée. C'est le clan maternel qui gère la propriété et les fruits du travail des

14 Cf. Jean-Paul DEMOULE, Les dix millénaires oubliés qui ont fait l'histoire-Quand on inventa l'agriculture, la guerre et les chefs, Paris, Fayard, 2017,176-180 ; James C. SCOTT, Homo Domesticus-Une histoire profonde des premiers Etats, Paris, La Découverte, 2019. 
champs. La force économique des femmes développe une forte solidarité entre elles. Cette solidarité est au fondement de la capacité à long terme qu'ont les sociétés matriarcales pour résister à l'influence patriarcale, comme cela est le cas en Indonésie des Minangkabau ${ }^{15}$.

Les sociétés matriarcales agraires ont précédé les sociétés nomades d'élevage. Les animaux ont d'abord été domestiqués dans les sociétés agraires. Les sociétés d'élevage se sont développées dans des zones où il n'était plus possible de pratiquer l'agriculture, mais où l'on pouvait encore faire paître les animaux. C'est pourquoi, à l'origine, les sociétés d'élevage étaient matriarcales. Quelques sociétés d'élevage matriarcales existent encore, chez les Tibétains d'Asie centrale, les Goajiro d'Amérique du Sud, les Bedja et Nubiens d'Afrique de l'Est et les Tonaregs d'Afrique du Nord. Contrairement aux sociétés patriarcales, les sociétés d'élevage matriarcales placent le soutien mutuel audessus de l'accumulation de biens, y compris des troupeaux. Ceci montre que l'élevage d'animaux ne mène pas en soi au patriarcat.

Dans les sociétés d'élevage matriarcal, les deux sexes jouissent de la liberté sexuelle. Le choix de l'époux revient aux femmes; le divorce et le remariage ont lieu facilement. Les femmes possèdent les tentes et les troupeaux, elles mènent une vie indépendante des hommes.

Quand les sociétés matriarcales doivent résister à l'envahissement de cultures patriarcales, elles ont tendance à développer une caste guerrière : Iroquois, Nayar d'Inde ${ }^{16}$, Ashanti, Touaregs. Lorsque ces sociétés doivent fuir les envahisseurs de sociétés patriarcales, notamment quand cela implique la migration de tout un peuple, elles procèdent parfois à une mainmise belliqueuse sur une autre société matriarcale. Se développe alors une société avec différentes classes matriarcales, comme dans le cas des Nayar et des Touaregs.

Les lieux de peuplements matriarcaux sont des villages-républiques autogérés qui ont les moyens de résister à la centralisation patriarcale. Cette résistance est fondée sur la gestion par les femmes des terres claniques et sur la façon dont les hommes défendent leurs sociétés matriarcales contre le monde extérieur. Il faut noter que les hommes défendent leur culture matriarcale, ce qui prouve qu'ils ne se sentent pas opprimés dans le matriarcat, contrairement à une vision ethnocentrique occidentale ou orientale (il y a aussi des cultures patriarcales en Orient et Extrême-Orient).

Sur le plan social, le clan est matrilinéaire : les enfants sont apparentés uniquement à leur mère et portent le nom de son clan. La plus jeune des filles hérite du clan de la mère les honneurs, les droits et les devoirs de responsable du clan. Ses frères l'aident et la protègent. L'un d'eux est choisi pour être leur

\footnotetext{
15 Cf. infra, p. 367 sq.

16 Cf.,infra, 368 sq.
} 
représentant délégué dans les conseils extérieurs au clan. Le clan est matrilocal: les enfants et petits-enfants, vivent avec leur mère dans la maison clanique.

La forme de mariage associée à de nombreuses cultures matriarcales est le mariage par groupes sœurs-frères, avec une prédominance de la polyandrie de frères pour les femmes dans certaines cultures spécifiques, comme au Tibet. Dans une société matriarcale matrilocale, ce sont les jeunes hommes qui effectuent un va-et-vient entre les deux clans. Les filles restent dans la maison clanique de leur mère.

Le mariage par groupes sœurs -frères a une signification économique : il empêche la division de la propriété et du cheptel. La polyandrie entre frères comporte également des principes écologiques. Elle engendre moins d'enfants et contrôle donc la démographie dans des sociétés où les ressources sont rares. Cette forme de mariage n'engendre ni conflit, ni jalousie. Ce type de mariage n'interdît pas aux partenaires de l'un ou l'autre sexe d'avoir leurs propres aventures sentimentales : ces liaisons sans lendemain ne fragilisent pas le mariage.

Les événements relatifs à l'amour et au mariage relèvent strictement du libre choix des femmes. La femme courtise l'homme, jamais l'inverse. Cette pratique peut prendre différentes formes : les visites, la cour, l'enlèvement.

Cette autorité des femmes n'engendre pas un système de domination de leur part. Un équilibre est maintenu entre les domaines d'activité des femmes et ceux des hommes. Ils sont définis différemment dans diverses sociétés, avec une marge de tolérance. Quand les femmes sont agricultrices, les hommes assurent les activités de pêche et de chasse. Les décisions sont prises par consensus.

En tant qu'époux et père, l'homme est un étranger dans la maison clanique de son épouse. Il n'est pas considéré comme apparenté aux enfants de celle-ci. Ses parents les plus proches sont les enfants de sa sœur ; il tient le rôle de père social vis-à-vis de ses nièces et de ses neveux. La sœur est sacrée pour son frère. Les deux relations affectives fondamentales sont donc celles unissant la mère et la fille, et la sœur et le frère. Toutes les autres relations affectives sont secondaires. Quand la paternité biologique est reconnue, le père prend soin des enfants de sa femme et participe à leur éducation. Cette attitude contraste avec la paternité patriarcale, axée sur la domination de l'homme sur les femmes et les enfants : le mari est le chef de famille, comme cela a longtemps été le cas en droit français.

Sur le plan politique, dans le cas où les conseils sont dirigés par des hommes, ils y siègent en tant que délégués envoyés par leurs clans. Ils rendent compte de leurs actes aux mères claniques et aux membres du clan. Cette charge de représentation est souvent confiée aux chefs ou aux rois. Ce sont les fils ou les neveux de la Grande Prêtresse. Cette dernière est la véritable détentrice de l'autorité ; les chefs ou les rois ne sont que ses délégués. Les mères claniques et les Grande prêtresse ne commandent pas par la force ; elles donnent des avis 
et des conseils. Quand il y a une prêtrise masculine, elle ne saurait exister sans une prêtrise féminine. Il n'existe pas de force de coercition : pas de police, ni de fonctionnaires, ni de soldats. Les chefs ou les rois ne peuvent lever l'impôt ; ils vivent aussi modestement que les autres membres de la tribu et peuvent être déchus de leurs fonctions et remplacés à tout moment. Il s'agit la plupart du temps, à proprement parler, de sociétés sans classes.

À l'heure actuelle, la majorité de l'humanité vit dans des systèmes patriarcaux, encore que le degré de domination des hommes soit différent suivant les sociétés, comme nous le verrons dans le cas des Han chinois. De plus, ils évoluent. Dans les pays occidentaux, depuis cinquante ans, les progrès des féminismes ont largement modifié la condition sociale et le statut juridique des femmes dans le sens d'un affaiblissement de la domination masculine.

Les systèmes matriarcaux passent pour une chimère, ou, au minimum, des résidus de l'histoire, victimes de la victoire du patriarcat et des Etats qui l'ont instauré. Rien moins que naturelle, la soumission des femmes pose problème. La biologie fait des femmes les principaux agents de la reproduction, un impératif majeur pour toutes les sociétés. Dans le passé, pour beaucoup d'entre elles, on ne soupçonnait pas le rôle de la fécondation masculine. Et non seulement, elles engendrent des femmes, mais aussi des hommes. Cela suscitait le trouble de certains philosophes, comme Aristote. Il en sortait en postulant que les filles avaient été engendrées par le testicule gauche, le côté gauche étant défavorable et de mauvais augure, les garçons par le testicule droit. Quoi qu'il en soit, ce privilège biologique aurait pu fonder une domination des femmes : elles sont la clé de la perpétuation de la famille. Catherine Capdeville note que justement elles ont été asservies en raison de ce privilège ${ }^{17}$.

Il faut y ajouter un autre argument. On a longtemps pensé qu'une des causes de la subjugation féminine était la moindre force physique. Il suffit de lire l'ouvrage d'Alain Testart sur l'anthropologie de la division sexuelle du travail ${ }^{18}$ pour s'apercevoir que les femmes sont capables de travaux exigeants sur le plan physique et qu'elles toujours participé à certaines activités cynégétiques. De plus, elles ne sont pas constamment enceintes ou obligées de s'occuper de nourrissons. Je n'ai pas la place pour étudier la véritable raison de tabous qui ont pesé sur les activités féminines, fondés sur certains particularismes physiologiques féminins, principalement les règles. Perdant périodiquement leur sang, les femmes ne peuvent pas être associées à certaines activités impliquant l'écoulement du sang : sang sur sang ne vaut.

17 Cf. Catherine CAPDEVILLE, Matriarcat et patriarcat en Chine- Han et Na, Langues Orientales, Inalco 2018, Les femmes à travers le monde, PP.14-19, Hal 02397136.

18 Cf. Alain Testart, L'Amazone et la cuisinière-Anthropologie de la division sexuelle du travail, Paris, Gallimard, 2014 
Mais ces arguments étaient ignorés ou oubliés : le patriarcat était dans la nature des choses. Du coup, les quelques auteurs qui s'intéressaient au matriarcat tombaient dans le même oubli ou étaient même discrédités ${ }^{19}$. Citonsen quelques-uns.

\section{b) Les recherches : des sociétés longtemps oubliées ou mécon- nues}

Johann Jakob Bachofen publie en 1861 Le droit maternel. En Europe, particulièrement en France avec le Code civil de Napoléon, c'était une époque particulièrement intense du patriarcat. Au XVIIIe siècle, dans l'aristocratie, il en allait tout autrement : dans les mœurs, l'activité des femmes était très supérieure à leurs droits. L'accession au pouvoir des bourgeoisies va leur être fatale jusqu'à la fin du XIXe siècle. Bachofen montre que la matrilinéarité n'était pas seulement une exception asiatique comme on le croyait à l'époque. On la trouve aussi en Inde, en Perse, en Égypte, en Méditerranée orientale. Il qualifie ces sociétés de sociétés de droit maternel(Mutterrecht). Mais il se trompe en les nommant gynocraties : rien dans les données qu'il cite n'autorise à penser à une domination des femmes. D'autre part, il subit l'influence de l'évolutionnisme, doctrine dominante à l'époque. Il imagine un premier stade de liberté sexuelle généralisée, qui aurait conduit à une révolte des femmes, par la suite victimes de la prise du pouvoir par les hommes.

Bachofen était en contact avec Lewis Henry Morgan qui étudiait en Amérique du Nord les Iroquois. Ce dernier constate également la matrilinéarité. Mais lui aussi l'intègre dans un schéma évolutionniste. La civilisation est apparue quand la propriété privée et la propriété foncière, domaines réservés des hommes, ont engendré une transformation des clans matriarcaux en clans patriarcaux.

Il est intéressant de noter que cet argument de la supériorité morale du patriarcat est encore utilisé aujourd'hui par certains Etats pour justifier leur domination sur les sociétés autochtones qu'il faudrait conduire, au besoin malgré elles, sur la voie du progrès.

Quelles sont les théories des auteurs marxistes de la même époque?

Pour Engels, ce qu'il croit être (à tort) la disparition des sociétés de droit maternel marque le début de la soumission des femmes, devenues des servantes et des esclaves sexuelles des hommes. Avec Marx, il pense que la monogamie est largement de façade : loin d'instaurer un système égalitaire entre le mari et son épouse, elle ne pèse en réalité que sur l'épouse. La monogamie n'est donc pas un signe de progrès. Quant à la monopolisation de la propriété privée par les

19 Cf. Heide GOETTNER-ABENDROTH, Les sociétés matriarcales-Recherchent sur les cultures autochtones à travers le monde les sociétés matriarcales-recherchent sur les cultures autochtones à travers le monde, Éditions des femmes, Paris, 2019,35-80. 
hommes, elle serait la conséquence de l'augmentation de la division du travail. Ce que ne corrobore pas l'observation empirique. Quelques cultures matriarcales urbaines font preuve d'une spécialisation du travail et d'une productivité élevée alors que la propriété privée n'est pas le monopole des hommes. Cependant, Marx et Engels sont également des auteurs évolutionnistes : il y a un sens de l'histoire, et l'apparition du patriarcat en est la manifestation.

Ces théories sont encore présentes dans certains Etats actuels. Il y a bien eu des sociétés matriarcales dans le passé. Celles qui subsistent à l'heure actuelle sont des sociétés arriérées et doivent être soumises à des mesures de développement.

On reconnaît un argument plus général des colonisations : le colonisateur apporte le progrès aux colonisés. Ce qui n'est d'ailleurs pas toujours faux, notamment dans le domaine de l'hygiène ou des techniques. Mais ces progrès s'insèrent dans une hiérarchie coloniale, qui fait des colonisés des inférieurs : l'égalisation des statuts ne peut être que postérieure au développement.

Tous ces auteurs travaillent à partir de sources de seconde main. Qu'en est-il des anthropologues ?

Bronislaw Malinowski a décrit les effets de la matrilinéarité à partir de ses recherches sur les habitants des îles Trobriand, en Mélanésie, en 1926. Il souligne le rôle considérable des femmes et, contrairement aux thèses évolutionnistes, affirme que les mœurs des Trobriandais sont sur bien des points supérieures à celles des civilisés.

Sur la question de la création des institutions permettant la vie en société, les opinions des anthropologues divergent.

En 1927 Robert Briffault publie un ouvrage monumental, The Mothers. Pour lui, les femmes sont les initiatrices de la société. L'autorité de la mère maintient la cohésion du clan des femmes et guide les fils dans leur mariage hors de celui-ci afin de créer des liens avec d'autres clans. La règle de l'exogamie provient des femmes, non des hommes. Mais ses travaux, qui ne correspondent pas à l'état social d'alors en Europe, sont méconnus.

Pour Claude Lévi-Strauss au contraire, la tribu a été une invention masculine. On n'est pas certain qu'il ait lu les travaux de Briffault.

En ce qui concerne la Préhistoire, on peut mentionner les travaux du paléo linguiste Richard Fester, en 1962 et 1974. Il estime que l'utilisation la plus ancienne d'outils n'est pas due à l'homme en tant que chasseur, mais aux mères s'occupant de leurs enfants. Ces outils leur ont servi à préparer la nourriture. Les noms des premiers outils dérivent dans la plupart des langues de racines nominales signifiant le féminin.

En 1970 les travaux de l'anthropologue David Jonas et de la biologiste Doris F.Jonas vont dans le même sens. Les femmes ont été déterminantes dans la formation des sociétés humaines dès le début de l'humanité et pendant des 
centaines de milliers d'années ensuite. Chez les primates, la femelle Alpha qui conduit le troupeau jouit du statut le plus élevé. Les mâles dominants sont exclus du centre du troupeau par leurs mères.

Mais les travaux de ces préhistoriens n'ont suscité aucune réaction.

Un seuil a cependant été franchi plus récemment.

Les recherches matriarcales féministes et autochtones, qui se recoupent sur bien des points, sont aujourd'hui en plein essor. La plupart du temps, les chercheurs sont des femmes. Ce qui comporte certains avantages. Elles sont en principe plus à même que les hommes de comprendre ce que signifie la maternité. Elles ont plus de facilité qu'eux à établir des contacts sur le terrain au sein des sociétés matriarcales existantes, cela d'autant plus lorsqu'elles sont autochtones .

Peggy Reeves Sanday a travaillé sur les Minangkabau de Sumatra, Heide Goettner-Abendroth sur les Mosuo de Chine . Ces derniers ont également été étudiés par une chercheuse elle-même Mosuo, Lamu Gatusa ${ }^{20}$. On pourrait citer d'autres exemples de chercheuses autochtones en ce qui concerne les Iroquois, le Ghana, la Kabylie.

Ces recherches contribuent à la lutte des peuples autochtones qui revendiquent leurs droits à avoir leur propre lecture de l'histoire et de la culture.

A été fondée en 1986 par Heide Goettner-Abendroth l'Académie internationale pour les Recherches matriarcales modernes et la spiritualité matriarcale. Elle a organisé plusieurs congrès internationaux, rassemblant pour la première fois des chercheuses venues d'Europe, des États-Unis et de Chine. Les chercheuses autochtones ont parlé non seulement des formes matriarcales existantes aujourd'hui dans leurs cultures, mais également des problèmes sociaux et politiques que la colonisation et l'influence des missionnaires ont apportés à leurs sociétés. Les Actes des deux congrès ont été publiés en 2009 en anglais dans l'ouvrage Societies of Peace, Past, Present and Future, Toronto, Inanna Publications, York University.

L'ouvrage de Heide Goettner - Abendroth, Les sociétés matriarcalesRecherchent sur les cultures autochtones à travers le monde (Éditions des femmes, Paris, 2019) rassemble des données extrêmement intéressantes sur les cultures matriarcales du passé mais aussi du présent, dans diverses régions du monde : la Chine, le Népal, la Corée, le Japon, l’Indonésie, la Mélanésie, la Polynésie, l'Amérique du Sud, l'Amérique centrale, l'Amérique du Nord, l'Inde du Sud, l'Afrique centrale, l'Afrique de l'Ouest et l'Afrique du Nord. Il est bien

20 Cf . LAMU GATUSA, Walk into the Women's Kingdom : Laku Lake, Mother Lake, Mosuo Women, Mosuo Daba Culture, Yunnan Academy for Social Sciences (en chinois uniquement) ; A Sacred Place of Matriarchy :Lugu Lake-Harmonious Past and Challenging Present, dans : Heide GOETTNER- ABENDROTH (dir.), Societies of Peace, Matriarchies Past, Present and Future, Toronto, Inanna Publications, York University, 2009. 
entendu impossible de résumer ici l'ensemble de cet ouvrage de plus de 500 pages.

En quoi cela intéresse-t-il le pluralisme juridique ? On sait que les Han sont l'ethnie dominante de la Chine. Or ceux-ci obéissent à un système patriarcal. On pense donc aux relations existant entre le système patriarcal Han et les systèmes matriarcaux de certaines minorités nationales.

Je privilégierai l'étude des $\mathrm{Na}$, une des minorités nationales du sud de la Chine.

\section{PARTIE II: TERRAINS : DROITS LOCAUX ET DROIT ÉTATIQUE}

J'étudierai d'abord la société $N a$ pour passer ensuite à l'étude des antagonismes entre le droit local $\mathrm{Na}$ et le droit central des gouvernements chinois. Je remarquerai ensuite que l'attitude des gouvernements chinois tendant à réduire le pluralisme juridique n'a rien d'exceptionnel.

\section{A) LA SOCIÉTÉ $N A^{21}$}

Le lecteur français regardera utilement sur Yu Tube le documentaire d'Arte de 52 minutes intitulé : Mosuo, le pays où les femmes sont reines.

\section{a) Les enquêtes du Pr CAI HUA à la fin du XX siécle}

Les $\mathrm{Na}$ ont été étudiés par CAI HUA, Professeur à la Beijing University, Directeur de recherches à l'Académie des sciences sociales du Yunnan. Il a obtenu un doctorat d'ethnologie à l'Université de Paris X, en France, en 1995.Ses travaux ont été lus par C.Lévi-Strauss et Francoise Héritier, qui l'ont encouragé dans ses recherches. Il a été accueilli au Laboratoire d'anthropologie sociale du Collège de France et le Laboratoire d'ethnologie et de sociologie comparative de l'université de Paris X- Nanterre. Les développements qui suivent sont largement repris de son ouvrage, dont j'ai résumé certains passages. Ne pouvant tous les citer précisément, j’ai indiqué en note la pagination des chapitres sur lesquels je me suis appuyé pour ces résumés.

Parmi les divers groupes que les Han appellent Moso, ceux qui habitent Yongning et ses alentours s'appellent eux-mêmes $\mathrm{Na}$. Agriculteurs montagnards, ils regroupent environ 30000 personnes.

Jusqu'à nos jours, femmes et hommes de cette ethnie pouvaient entretenir librement des relations sexuelles avec plusieurs partenaires et en changer selon leur gré.

Leurs mœurs ont toujours semblé très étranges aux Han.

21 Cf. CAI HUA, Une société sans pére ni mari-Les Na de Chine, Paris, Presses Universitaires de France, 1997. 
Li Jinq, auteur de la dynastie Yuan (1279-1368), note dans son Récit monographique du Yunnan: "les femmes ne se sentent pas honteuses de laisser apercevoir leur sexe. Une fois mariées, elles n'ont plus de tabous sexuels"

Sous la dynastie des Ming (1368-1644), Xie Zhaozhe remarque que mari et épouse ne se voyaient pas pendant la journée mais partageaient la même couche la nuit. Les enfants ne pouvaient voir leur père avant l'âge de dix ans. L'épouse et les concubines n'étaient pas jalouses les unes des autres. Il se demandait pourquoi le père n'éprouvait pas l'envie de voir ses enfants et s'étonnait de l'absence de jalousie.

Les Occidentaux partagent l'étonnement des Chinois. Marco Polo écrit que les Moso n'ont pas honte de leurs coutumes, même si le Grand Khan les a interdites.

Plus près de nous, en 1947, J.F. Rock, un anthropologue américain, note que leurs coutumes sont l'inverse de celle des Chinois et que la promiscuité sexuelle entraîne un nombre très élevé de cas de syphilis et de maladies vénériennes. Il juge que les normes de moralité ne sont guère élevées ${ }^{22}$.

Pour disposer d'études sérieuses sur les $\mathrm{Na}$, il a fallu attendre jusqu'aux années 1960. Entre 1956 et 1963, le Comité permanent de l'Assemblée populaire nationale de Chine a lancé des enquêtes sur toutes les ethnies minoritaires du Yunnan. Un premier travail systématique sur le terrain a été effectué en 1960 par trois chercheurs chinois : Song Enchang, Zhu Baotian, Wu Guanghu. Une dizaine d'ethnologues sont revenus sur le terrain de l'hiver 1962 au printemps 1963 et ont publié un rapport d'enquête en quatre volumes. Issus de ces enquêtes ont été publiés deux ouvrages disponibles seulement en chinois : Zhan Chengxu, Li Jinchun, Wang Chengquan, Liu Longchu, Yongning Naxi Zu de Azhu Hunyin he Muxi Jiating(Le mariage d'azhu et la famille matrilinéaire chez les Naxi du Yongning),1980, 321p. ; Yan Ru-xian et Song Zhao-lin, Yongning Naxi Zu de Muxi Zhi, ( Le systéme matri linéaire des Naxi de Yongning), Kunming, Editions du Peuple du Yunnan, 1983, 449 p.

Leurs analyses ont été faites suivant les critères du marxisme, marqué, nous l'avons vu, par l'évolutionnisme. Ils considéraient qu'il était donc normal qu'il subsiste dans les rapports matrimoniaux des résidus du mariage par groupe et des traces de la communauté clanique matrilinéaire .Entre 1970 et la fin du $\mathrm{XXe}$ siècle, des articles complémentaires sont parus, qui ne présentent pas de divergences fondamentales par rapport aux travaux précédents.

Le Pr.Cai Hua fait remarquer ${ }^{23}$ que les enquêteurs ne parlaient pas la langue $\mathrm{Na}$ et sont donc passés à côté de données fondamentales, ce qui minore la valeur de leurs enquêtes.

22 Cf. J.F. ROCK, The Ancient Na-Khi Kingdom of Southern China, Harvard, 1947, vol.2, p 391.

23 Cf. CAI HUA, op.cit, 19. 
Son premier séjour sur le terrain a eu lieu durant l'été 1985. Il y est retourné un an plus tard. Il s'intéressait particulièrement aux modalités de la visite et de la cohabitation. Il se demandait aussi pourquoi les $\mathrm{Na}$ vivaient sans mariage, une exception dans la littérature anthropologique. Son troisième séjour, d'une durée d'un an, a eu lieu entre 1988 et 1989. Le quatrième séjour a eu lieu en juillet 1992.

Les témoignages rapportés par le Pr. Cai Hua sur les réactions de la population par rapport aux résultats des enquêtes sont extrêmement intéressants ${ }^{24}$ :

"Fiers de leurs coutumes, les Na en parlent aisément aux chercheurs arrivés sur le terrain dans les années 1960. Les publications de ces chercheurs ont valu bien des problèmes aux Na, surtout la "Campagne de monogamie " pendant la Révolution culturelle qui avait pour but de les normaliser de force. "Chat échaudé craint l'eau froide ! ». Depuis lors, en ce qui concerne leur tradition, ils évitent soigneusement de la laisser entrevoir à ceux qui sont étrangers à leur communauté (...) Les gens de Yongninq surveillent les étrangers, surtout les enquêteurs. À chaque fois que quelqu'un y arrive en vue d'une enquête sur leurs coutumes, ils mettent tout de suite leurs responsables au courant. Notre arrivée pour la première fois n'a pas fait exception. Lorsque nous avons rencontré un des responsables $\mathrm{Na}$, il nous a averti d'emblée : " Les enquêteurs des années 1960 ont publié des racontars sur nos coutumes, ils ont déformé les faits ». Il a ajouté : "Les gens ont déjà dit clairement que, s'ils remettent les pieds à Yongning, ils les tueront. Si jamais cela arrive, nous n'y pourrons rien".

Nous avons essayé de leur expliquer que la théorie ayant guidé ces premiers chercheurs s'appelle l'évolutionnisme et que notre point de vue différait. Si les précédents enquêteurs n'avaient pas compris ce qu'est leur tradition, ce n'est qu'une raison de plus pour entreprendre de nouvelles études afin qu'on connaisse la réalité. Après une longue discussion nous avons réussi à les convaincre. [En 1988] j'ai été reçu dans la maison d'une lignée dont la maîtresse pratique la cohabitation avec un cadre $\mathrm{Na}$ en retraite. Cette lignée est composée de deux générations. Au bout de quatre mois et demi environ, ce dernier m'exprima ses inquiétudes:

«Tu viens enquêter chez nous. Nous voulons bien te soutenir. Mais si jamais un jour tu publies ton étude, et que des problèmes surviennent, comme ce fut le cas après l'enquête des années 1960, nous serons seuls compromis: les gens vont dire que tu habitais chez notre lignée, nous accusant de t'avoir tout raconté. Nous ne pouvons pas prendre ce risque. Ce serait mieux pour nous que tu changes de maison maintenant. De la sorte, nous ne serons pas seuls en cause».

En effet, l'organisation familiale Na suscitait l'incompréhension des Han. 


\section{b) L'organisation familiale ${ }^{25}$}

Pour les $\mathrm{Na}$, ong, l'os, est traditionnellement considéré comme le vecteur des caractères héréditaires. Il vient de la mère. À sa naissance, un enfant fait automatiquement partie du groupe de sa mère. Ceux qui ont le même ancêtre féminin résident sous le même toit à chaque génération. Les sœurs et les frères travaillent, consomment, élèvent ensemble les descendants issus de celles-là. Ils résident ensemble pendant toute leur vie. Le groupe de parents résidentiels n'est donc composé que de consanguins, et est exclusivement matrilinéaire.

Au sein de chaque matrilignée, deux chefs :un homme et une femme. Le chef féminin s'occupe des affaires intérieures de la maisonnée : distribution des vêtements, gestion des réserves et des dépenses, organisation du travail au foyer et aux champs, préparation des repas quotidiens. Le chef masculin se charge de contacter les étrangers pour tout ce qui touche à la terre, au bétail et aux entraides temporaires ou annuelles .Dans certaines lignées, c'est la femme chef qui domine, dans les autres, l'homme chef.

Tout ce qui touche aux relations sexuelles et sentimentales est soumis à une obligation de discrétion : c'est la moralité $\mathrm{Na}$.La liberté des mœurs est donc encadrée et n'a rien à voir avec la débauche.

Il est strictement interdit de parler de relations sexuelles, affectives et sentimentales en présence des consanguins des deux sexes. Quand l'oncle maternel a une remarque à faire à sa nièce d'ordre sentimental, il ne peut la confier qu'à un étranger pour la lui faire connaître. En revanche, si les mères ont des remarques à faire à leurs fils, elles peuvent leur parler de façon discrète, mais directement. Il est interdit d'aller se baigner en même temps dans la source thermale. On ne peut danser côte à côte. Il est strictement interdit de dire des mots grossiers et obscènes. On ne peut se mettre côte à côte au cinéma, même pas dans le même rang. Car à l'écran peuvent surgir des images à caractère affectif. Devant la télévision, ne sont assis que des membres d'un même sexe. Tout se passe comme s'il y avait une séance pour les hommes et une autre pour les femmes. Il est interdit de faire chemin ensemble pendant la nuit. Lorsque des consanguins de sexe différent sont témoins d'une dispute d'un couple d'une autre ethnie dans un village, ils doivent s'en éloigner tout de suite, car pendant la querelle, les protagonistes risquent de prononcer des mots grossiers. La règle vaut également dans une querelle entre deux villageois de même sexe.

En Europe occidentale, la relation fraternelle est rarement sanctionnée par le droit. Il n'existe pas d'obligation naturelle à l'intérieur d'une fratrie. Le droit porte plutôt son attention sur les relations entre parents et descendants et entre époux. Au contraire, chez les $\mathrm{Na}$, la relation sororale est très importante ${ }^{26}$.

25 Cf. CAI HUA, op.cit., 97-103.

26 Cf. CAI HUA, op.cit., 115-117. 
Si on compare avec les autres sociétés, la place de la relation mari/femme est remplacée en très grande partie par la relation sœur/frère. Elle comporte deux aspects. Le premier est celui de la camaraderie plus ou moins intime pendant l'enfance, qui s'accompagne d'un développement progressif d'un tabou contre l'inceste s'accompagnant d'une certaine réserve et de la spécialisation économique. Le second aspect, lorsqu'ils sont devenus adultes, est celui de la responsabilité conjointe quand aux soins matériels et affectifs à donner aux enfants de la sœur ; la coopération économique, l'égalité des droits en ce qui concerne la propriété et les sphères respectives d'autorité.

Sur le plan résidentiel, étant donné l'institution des visites, sœurs et frères ne se séparent pas, malgré les tabous contre l'inceste. Il y a là une différence avec la relation mari/femme. Entre mari et femme existent les relations sexuelles ; mais ils sont séparables par le divorce. À l'inverse, entre frères et sœurs il n'y a pas de commerce sexuel, mais ils sont inséparables pendant toute leur vie.

À la place des relations père/fils et père/fille existant dans notre société, chez les $\mathrm{Na}$ ce sont celles oncle maternel/neveu et d'oncle maternel/nièce. Le rôle joué par le pére beaucoup d'autres sociétés est ici tenu par l'oncle maternel.

Des scissions peuvent cependant se produire.

Quand il y a dissension entre une femme et le reste de sa matrilignée, la femme quitte sa maison d'origine pour s'installer ailleurs, en général dans le même village. Elle ne conserve qu'un petit terrain pour construire sa maisonnette, quelques ustensiles de cuisine et quelques instruments agricoles simples. Les $\mathrm{Na}$ appellent cette scission baolu, ce qui signifie "construction d'une petite maison ". Cette scission ne concerne que les femmes. Lorsqu'un garçon ne s'entend plus avec sa matrilignée, il doit s'expatrier et commencer une nouvelle vie ailleurs, car pour les $\mathrm{Na}$, un garçon ne peut s'en sortir tout seul.

Un deuxième type de scission constitue à diviser la maisonnée et partager le patrimoine. Il peut avoir lieu pour plusieurs raisons : dissensions entre frères et sœurs, dissensions entre deux sœurs, naissance de filles en surnombre.

\section{c ) Les modalités des relations sexuelles ${ }^{27}$}

Il existe quatre modalités de relations sexuelles, bien décrites par le Pr Cai Hua. La visite furtive (nana sésé), la visite ostensible (gepié sésé), la cohabitation d'un couple sans banquet rituel( tidzi-ji mao the), la cohabitation après le festin( tidzi ji the).

La modalité la plus pratiquée est la visite furtive. Nana signifie furtivement. Nous l'étudierons en premier.

27 Cf. CAI HUA, op cit, 143-250. 


\section{1) LA VISITE FURTIVE ${ }^{28}$}

C'est la pratique la plus répandue. .L'expression indique une rencontre galante à la dérobée ou une visite furtive qui se déroule sans que ne le sachent les consanguins, notamment les adultes masculins de la visitée. Une femme et un homme qui établissent cette relation s'appellent discrètement entre eux : açia. Les deux açia sont considérés comme étrangers l'un par rapport à l'autre ; leur relation est strictement d'ordre privé. C'est une relation uniquement sentimentale, amoureuse et sexuelle. La relation d'açia commence à 15 ans pour les filles, 17 pour les garçons. Chez les $\mathrm{Na}$, l'activité sexuelle se situe entre 13 et 55 ans pour une femme, 13 et 61 ans pour un homme. Continuer la vie sexuelle après ces limites d'âge est désapprouvé, mais pas interdit. La virginité n'a aucune importance.

Une telle relation peut durer entre une nuit et quelques années. D'ordinaire, la visite de l'homme s'effectue dans la chambre de la femme aux environs de minuit ; le visiteur quitte la femme à l'aube pour regagner sa maison. Femmes et hommes sont en situation d'égalité. Une femme aussi bien qu'un homme peut prendre l'initiative des avances. Le rendez-vous peut se prendre après un temps de réflexion, ou bien immédiatement, à condition que cela s'effectue en l'absence de tout consanguin de sexe opposé de deux intéressés. Les rendez-vous sont fixés dans la journée, au moment qui paraît le plus propice. Aujourd'hui, le cinéma est un lieu qui convient parfaitement à ce genre de flirt, parfaitement accepté par les filles : le film a peu d'importance .Si les partenaires sont consentants, à la sortie du cinéma, ils vont passer la nuit ensemble. Pendant la rencontre, ils doivent parler à voix basse, afin que rien ne parvienne aux oreilles des consanguins de la femme. Toute rencontre est interdite pendant la journée. Il est interdit aux femmes de rendre visite aux hommes. Les $\mathrm{Na}$ font remarquer qu'ils ne sont pas comme les Han, parmi lesquels les ascendants cherchent toujours à arranger les rencontres : ils sont totalement libres.

Quels sont les critères de choix ?

Les femmes privilégient la beauté physique ; ensuite, l'humour et la capacité au travail ; enfin la tendresse et la générosité. Pour les hommes, le critère essentiel de choix repose sur la beauté. Plus une femme est belle, plus le nombre de ses soupirants augmente. Les femmes qui sont laides ou handicapées ont très peu de chances d'être visitées ; à partir de trente ans, les femmes sont beaucoup moins sollicitées. Au bout d'un certain temps, si les visites du même homme se multiplient, la fille peut en parler avec sa mère. Mais la mère n'a pas de pouvoir de décision en ce qui concerne le maintien ou la cessation de la

28 Cf. Pascale-Marie MILAN, Tourisme et changement social chez les Na de Chine-Etude comparée d'une coutume sexuelle : le séssé, Thèse Anthropologie, Université Laval, Québec, Canada ; Université Lyon 2, Lyon, France, 2019. 
relation. La liaison est purement sentimentale et sexuelle, mais il arrive que les hommes laissent un peu d'argent lors de leurs visites. De manière générale, il y a peu de cadeaux matériels entre les partenaires.

Un individu peut entretenir des relations simultanées avec plusieurs açia. Une femme peut recevoir deux ou trois visiteurs par nuit ; un homme peut rendre visite à deux ou trois femmes pendant la même nuit. Même si certaines relations durent longtemps, chacune d'entre elles n'est conçue que dans le présent : la notion d'engagement pour le futur n'existe pas. Il est très facile de rompre la relation. Une femme qui veut la terminer dit à son partenaire de ne plus revenir ; un garçon n'a même pas besoin d'expliquer la raison de sa décision de mettre fin à la relation. D'ailleurs, le cas d'un individu n'ayant établi qu'une relation d'açia pendant toute sa vie n'existe pas. Un homme beau et/ou riche qui n'a connu que quelques açia risque d'être tenu pour inapte et maladroit.

Fait très étrange pour les observateurs étrangers depuis des siècles, la jalousie n'existe pas. Cela tient au fait que pour les $\mathrm{Na}$, le serment de fidélité est honteux, car il est conçu comme un négoce, un échange qui n'est pas conforme aux coutumes. Aucune relation sexuelle ne peut mener les amants à se promettre le monopole de la sexualité. La sexualité n'est pas un marchandage, mais une affaire purement amoureuse et sentimentale qui n'implique aucune contrainte mutuelle. C'est une différence essentielle par rapport à nos sociétés, dans lesquelles l'engagement de fidélité est tenu comme la preuve de l'authenticité de la relation. Les $\mathrm{Na}$ disent que s'ils arrivent chez une femme qui est déjà prise, ils ne sont pas jaloux. Les femmes ne manquent pas, il suffit d'aller voir ailleurs. Un proverbe $\mathrm{Na}$ dit : "Derrière chaque versant de montagne, existe un village, où n'y a-t-il pas de femmes ? ". Une femme ne doit rien à un homme, il n'y a donc pas de raisons d'être jaloux.

Bien entendu, à la suite de ces relations, certaines femmes peuvent tomber enceintes. En ce qui concerne la filiation, c'est à la ressemblance physique de l'enfant à un des açia de la femme que les $\mathrm{Na}$ jugent de qui est issu cet enfant. Si aucune ressemblance physique n'est constatée, ils considèrent que le géniteur est celui qui fréquentait la mère à la période où elle est tombée enceinte si, à ce moment-là, il était le seul visiteur. La question est d'ailleurs de relativement peu d'importance : il importe peu de savoir qui est le géniteur de qui. En général, une femme ne dit pas à son enfant qui est son géniteur, un homme ne dit pas un enfant qu'il est son géniteur. Même s'il le sait, un enfant, garçon ou fille, ne dit jamais qui est son géniteur, tout cela à cause de l'interdiction d'évocation sexuelle. Le terme de bâtard n'a aucune connotation péjorative. Que le géniteur soit connu ou ignoré n'a aucun effet sur le statut de l'enfant. Tous les enfants sont traités de la même manière au sein de la lignée aussi bien que dans la société. Dans l'enquête menée par le Pr. Cai Hua, 14,05 \% de l'échantillon d'enfants étaient de géniteurs inconnus. 


\section{2) LA VISITE OSTENSIBLE}

A la différence de la relation furtive, elle n'est pratiquée que par une minorité de de $\mathrm{Na}$.

L'expression désigne la visite qu'un homme rend ostensiblement à une femme sans qu'il ait à se cacher des membres de la lignée de celle-ci. Ceux qui pratiquent les visites ostensibles ont toujours commencé par les visites furtives. À mesure que les sentiments qu'ils éprouvent l'un pour l'autre s'approfondissent, les deux partenaires échangent leurs ceintures, inscrivant ainsi dans la durée leurs sentiments. Entre eux ils ne s'appellent plus simplement $a c ̧ i a$, mais $d b u z i$ $d h u m i$, ce qui signifie amis, ou partenaires. Une fois que les deux partenaires s'entendent pour modifier leurs relations, la femme en parle avec sa mère pour envisager le jour où l'homme pourra effectuer une visite officielle dans la maison. Quand l'homme connaît bien la lignée de la femme, il peut venir seul. Sinon, il se fait accompagner par un moio, un intermédiaire. C'est toujours l'homme qui choisit l'intermédiaire. Si c'était une femme, elle serait tenue pour « une truie en chaleur qui fonce dans le brouillard".

La rencontre a lieu dans la salle principale, vers 11 heures du soir, en l'absence des membres masculins de la lignée. Elle est suivie d'un repas rituel. Avant les années 1960, l'homme offrait au moins une veste à la femme, cette dernière lui donnait un pantalon. À partir de cette rencontre avec le chef féminin de la lignée, l'homme aura tendance à arriver un peu plus tôt et un peu moins discrètement lors de ses visites nocturnes.

Les partenaires à la relation ouverte restent cependant étrangers l'un à l'autre, comme dans la visite furtive. Si la relation engendre des enfants, l'homme n'est soumis à aucune obligation économique ou juridique à leur égard. Il n'a non plus aucun pouvoir sur eux. Devenus adultes, les enfants n'ont pas le devoir de s'occuper de leurs géniteurs pendant sa vieillesse.

Un autre trait de la visite ostensible est que les deux partenaires se doivent maintenant l'un à l'autre le privilège des relations sexuelles. En règle générale, une personne ne vit en relation ouverte qu'avec une seule autre.

Cependant, dans la pratique, cela n'entraîne pas l'abandon des rapports d'açia. La femme peut continuer à recevoir des visiteurs furtifs, ce qui est désapprouvé par les coutumes, mais pas interdit. Pratiquer l'amour furtif avec une personne en relation ouverte est qualifié par une expression qui signifie " voler le sexe ". Une femme enceinte peut continuer à recevoir des visites, qu'elle vive en relation ouverte ou furtive.

Cela peut évidemment entraîner des conflits à la fois entre les partenaires à la visite ostensible, et entre l'homme et son ou ses rivaux furtifs. En fait, la continuation de relations furtives n'est pas blâmée tant qu'elle n'est pas connue de l'autre partenaire : dans la pratique, la liberté sexuelle domine. Ce n'est que 
lorsque surgissent des conflits que survient la notion de privilège sexuel. Entre les partenaires, si la relation d'açia prédomine dans leur esprit, c'est la tolérance qui réglera leur attitude. Si leur désir de possession exclusive l'emporte, la jalousie surviendra. L'arbitre est alors la société. Si l'exclusivité est revendiquée comme un droit réel au travers de réactions vives de jalousie, la moquerie rappellera le jaloux au principe de liberté sexuelle. Si la jalousie engendre des violences, elle sera considérée comme une infraction pénale.

Mais les partenaires à la visite ostensible ne peuvent en aucun cas s'imposer l'un à l'autre : la décision d'un seul suffit pour rompre la relation. Celui ou celle qui voudrait instituer un monopole est considéré comme adoptant un comportement honteux. En général, les hommes abandonnent leurs partenaires plus fréquemment que les femmes. La rupture intervient souvent lorsque l'homme est attiré par une autre femme. La relation ne dure qu'autant que des sentiments mutuels existent entre les deux intéressés.

\section{3) LA COHABITATION}

Ti dzi ji mao the qualifie cette troisième modalité. Elle signifie au sens propre s'asseoir, au sens figuré s'installer. L'expression indique une relation dans laquelle une personne est venue s'installer chez son ou sa partenaire sans inviter de convives à un festin. C'est ce qu'on l'on peut traduire par cohabitation. Il existe trois types de cohabitation : uxorilocale, virilocale, néolocale.

La plupart des cas uxorilocaux apparaissent dans la lignée où la main-d'œuvre masculine fait défaut. La cohabitation a pour but d'assurer la subsistance de la maisonnée. Par exemple, lorsqu'une lignée ne compte que des filles ou une fille unique dans la jeune génération. La compagne n'est pas considérée comme une parente par la lignée de son partenaire. Sa situation n'est donc pas comparable à celle de la belle-fille dans beaucoup d'autres sociétés.

La virilocalité apparaît dans la situation symétrique inverse : dans les lignées où il n'y a que des fils, ou un fils unique. De manière identique, le compagnon n'est pas tenu pour un parent par la lignée de sa partenaire. Ce n'est donc pas un gendre.

La néo localité débute par une virilocalité. Les hommes qui ont amassé un certain patrimoine font en sorte que leur partenaire cohabite dans leur maison d'origine. Cela peut provoquer des conflits entre les mères et les sœurs de l'homme et sa partenaire, ce qui provoque parfois des scissions.

Dans tous les cas, pour qu'une personne puisse s'établir chez son ou sa partenaire une condition doit être remplie : sa maisonnée doit être nombreuse, de sorte que son départ n'entraîne aucun manque significatif

Sans exception, la cohabitation est précédée par une relation furtive et/ou par une relation ouverte. La personne ayant eu le consentement de sa lignée part seule. Elle emporte ses habits, ses affaires personnelles; les membres de sa lignée 
ne l'accompagnent jamais ; la lignée d'accueil n'envoie aucune compensation matérielle. En général, une lignée n'admet pas que des individus d'une autre consanguinité que la sienne s'installe sous son toit si elle ne manque ni de membres féminins, ni de membres masculins.

Si c'est une femme qui part, elle jouera le rôle de chef dans sa nouvelle maisonnée, l'homme ayant un rôle inférieur. Toutefois, la femme n'a pas le droit de présider les rituels, signer les contrats de gros achats ou ventes, même si son avis pèse lourd dans ces transactions. Si c'est l'homme qui part, il assumera davantage de travaux que lorsqu'il était dans sa propre maisonnée. Il sera perçu comme un domestique de la maisonnée d'accueil.

Les deux cohabitants doivent respecter un privilège sexuel mutuel. Toutefois, comme dans la relation précédente, dans la pratique, cette condition n'est pas toujours respectée. Les partenaires peuvent donc entretenir parallèlement à la relation de cohabitation des relations d'açia. Mais concrètement, puisqu'il y a cohabitation sous un même toit, les conditions de discrétion sont beaucoup plus difficiles à réunir.

Au sein de la maison d'accueil, les cohabitants sont respectivement assimilés à la sœur et au frère de leurs partenaires du point de vue de la terminologie de parenté. Les deux partenaires sont considérés comme amis et étrangers l'un par rapport à l'autre. Ces valeurs concernant la position des cohabitants sont demeurées les mêmes jusqu'à nos jours.

Après la survenue d'enfants, quand la relation de cohabitation n'est pas dissoute, les enfants résident avec leur mère dans la lignée d'accueil, portent le nom de la lignée d'accueil. Dans le cas de cohabitation néolocale, les enfants portent le nom de la nouvelle maisonnée. Ce nom peut être aussi bien celui de l'homme que celui de la femme.

Les cohabitants possèdent la liberté de rompre la relation, quand ils le souhaitent, sans aucune formalité ni rituel. Lors de la rupture de la relation, en cas de virilocalité ou de courte durée, la femme a le droit d'amener tous les enfants, mais pas les biens. En cas de longue durée, la lignée d'accueil a le droit de garder des enfants, et la femme a le droit sur certains biens mineurs. En cas d'uxorilocalité, quelle que soit la durée, l'homme n'a de droit ni sur les enfants ni sur les biens.

\section{4) LE MARIAGE DU FILS}

Le mariage suppose des prestations matérielles importantes; ce sont donc uniquement les familles riches qui adoptent cette modalité de relations.

Cette modalité est principalement associée au mode de résidence virilocal rarement à l'uxorilocalité. Comme dans la cohabitation, la cause principale du mariage est le manque d'un représentant d'un sexe à une génération donnée. D'autres facteurs jouent également : un conjoint d'un couple et d'une autre ethnie 
ayant une autre tradition matrimoniale, conjoint commerçant et fréquentant d'autres lieux, régis par d'autres coutumes.

Quand la lignée du fils a fixé sa préférence pour une femme d'une autre lignée, elle envoie son chef féminin ou masculin discuter avec le chef féminin ou masculin de l'autre lignée. Cette autre lignée doit posséder beaucoup de filles, sans quoi elle ne donnerait jamais son accord. Ensuite, une date est fixée pour la première intervention de l'entremetteur. Celui-ci apporte un certain nombre de cadeaux, qui sont offerts à la lignée de la fille. Les négociations se poursuivent quant au prix de la fille, qui varie en fonction de la richesse, du statut social des deux lignées et de la beauté de la fille. La virginité de la femme n'a aucune importance. Elle n'intervient pas dans le critère de choix de l'épouse. Si l'épouse a eu d'autres enfants avant le mariage, ceci ne constitue pas une gêne pour le mariage.

Ensuite, c'est le festin de noces. Pendant les noces, l'épouse n'est jamais aux côtés de l'époux devant le public. Pendant le banquet, les mariés mangent isolément, chacun de leur côté. Après le mariage, ils n'ont pas d'emplacement commun pour dormir. Quand ils ont envie d'être ensemble, c'est au mari de venir rejoindre son épouse, jamais l'inverse. Les deux partenaires sont devenus mari et épouse. L'épouse travaille avec toute la lignée de son mari. Si le mariage a été uxorilocal, il y aura beaucoup moins de travaux à la charge du mari. Les couples mariés ne se prennent jamais en photo ensemble. Le mari et l'épouse vivent sur un pied d'égalité.

En cas de querelle, l'épouse s'échappe chez sa mère. Si le conflit s'aggrave et que l'un désire abandonner l'autre, et que ce dernier n'accepte pas la séparation, les représentants de chaque lignée du village vont s'efforcer de les réconcilier. Si le conflit malgré tous s'aggrave, les représentants vont alors intervenir pour juger du partage des enfants et des biens.

Dans la plupart des cas la relation de couple est précédée de relations furtives et/ou ouvertes. En général, le mariage ne met pas fin aux relations furtives avec d'autres partenaires : la condition en sera toujours la discrétion.

La dissolution de la relation matrimoniale peut prendre deux formes : la fuite furtive, l'abandon. La fuite intervient en général en cas de mariage arrangé qui tourne mal.

Les conditions de dissolution du mariage révèlent la finalité de ce type d'union. Quand le mariage a échoué peu de temps après la noce à cause de l'inadaptation de l'épouse, sa lignée a l'obligation de restituer la prestation pour que la lignée preneuse puisse renouer l'union avec une autre lignée. Lorsque le divorce, au contraire, a lieu après de longues années de mariage, le mari a le droit d'obtenir des enfants au lieu de rendre la prestation. Plus la vie commune a été longue, plus l'épouse obtiendra de biens; plus ils ont eu des enfants, plus 
le mari en obtiendra. Dans les deux cas, les règles sont conçues pour assurer la descendance de la lignée preneuse.

La cohabitation et le mariage sont des solutions remédiant au manque de représentant féminin dans une génération donnée. Mais pourquoi choisir l'une plutôt que l'autre, ou l'inverse ? Pour une lignée manquant de descendance féminine, il faut absolument que vienne une femme. Si elle est riche, la maisonnée optera plutôt en faveur du mariage, qui est une solution plus sûre. Si une lignée manque de descendance masculine, le mariage n'est pas indispensable : elle peut avoir des enfants par des visiteurs. Il lui suffit de choisir la cohabitation pour résoudre le problème de la main-d'œuvre. C'est pourquoi il y a un pourcentage plus fort de cas uxorilocaux dans les cas de cohabitation.

\section{d) Que nous dit sur le mariage la pré-éminence de la visite furtive ? ${ }^{29}$}

Le Pr.Cai Hua insiste sur le fait que la visite est extrêmement différente du mariage et que cet exemple d'une société établie sur une institution autre que celle du mariage est unique dans la littérature anthropologique.

Dans les sociétés non matriarcales, en particulier celles qui connaissent une règle de transmission patrilinéaire, l'institution du mariage est indispensable. Sans elle, il serait difficile à un homme d'avoir des droits sur les enfants dont il est le géniteur. En revanche, lorsque la règle est matrilinéaire, une société peut se passer du mariage. Mais la plupart des sociétés matrilinéaires ont cependant instauré le mariage. Comme on l'a vu, la différence essentielle entre le mariage et la visite tient à ce que dans le mariage les partenaires s'appartiennent et doivent collaborer sur le plan économique, alors que dans la visite, il n'y a pas de privilège sexuel ni de coopération économique. De plus, dans le mariage, en règle générale, les partenaires vivent sous le même toit, ce qui n'est pas le cas dans la visite.

Le Pr.Cai Hua pense que dans la nature humaine, il y a à la fois un désir de possession du partenaire, mais aussi celui de la multiplication des partenaires. Pour lui, ce sont deux invariants. Le désir de possession, d'exclusivité, se retrouve dans l'institution du mariage. Mais d'un autre côté, on observe que le sentiment passionnel s'épuise avec le temps et que tout être humain peut-être attiré par plusieurs autres individus, même simultanément, et pas seulement par un seul. Tout le monde sait, en Chine comme en France, qu'on peut tomber amoureux plusieurs fois. Du désir de diversité naît donc le désir de multiplication des partenaires.

Sur le plan théorique, la seule formule susceptible de répondre simultanément à ces deux tendances serait une institution dans laquelle un

29 Cf. CAI HUA, op.cit., 323-331,335-340,356-361. 
nombre égal d'hommes et de femmes se posséderait simultanément. Mais on n'en trouve aucun exemple dans la littérature anthropologique. Cette modalité est en effet en elle-même contradictoire. Elle débouche non sur la possession, mais sur le partage. Ne restent donc que deux possibilités : la possession sans jouissance de la diversité, ou bien la jouissance de la diversité sans la possession. On retrouve ces deux formules d'une part dans le mariage, d'autre part, dans la visite. Mais le désir de la diversité se manifeste aussi dans le mariage par l'existence de l'adultère, très fréquent, même s'il est juridiquement condamné. Ce qui entraîne dans les sociétés à mariage la multiplication des divorces, que nous connaissons bien dans nos sociétés contemporaines. L'intérêt de la modalité de la visite est qu'elle concilie la liberté, mais qu'elle peut aussi s'inscrire dans la durée, puisque la visite peut durer plusieurs années.

Le Pr.Cai Hua insiste aussi sur l'idée que contrairement à une opinion courante en anthropologie, l'instauration de l'institution du mariage n'est pas la seule à garantir la reproduction humaine : la visite remplit également cette fonction.

Il fait également remarquer que la visite débouche sur une mise en cause de la théorie de Lévi-Strauss. Celui-ci a écrit que le point central de la parenté est l'échange institutionnalisé des femmes par l'alliance du mariage. Il estime aussi qu'en raison de son universalité, la prohibition de l'inceste nécessite le mariage, que la division du travail entre les sexes rend également le mariage indispensable, et que, sans mariage, aucune société ne pourrait se maintenir ${ }^{30}$. Le mariage serait donc consubstantiel à l'humanité. À l'inverse, la société $\mathrm{Na}$, dans laquelle le mariage est peu pratiqué, est l'exemple d'une société dans laquelle, dans l'institution de la visite, existent aussi la prohibition de l'inceste, et la division sexuelle du travail .Le tabou de l'inceste, dans n'importe quelle société, n'interdit directement que le rapport sexuel, non pas le mariage. L'exemple $\mathrm{Na}$ montre donc que sans mariage, une société peut parfaitement se maintenir et fonctionner aussi bien qu'une autre.

N'est-ce pas d'ailleurs ce que nous dit l'évolution actuelle des sociétés occidentales, dans lesquelles les mariages diminuent, sans que cela mette en cause leur existence ? Une solution juridique serait peut-être le mariage à durée déterminée, que connaissent bien les musulmans chiites, qui permet à la fois l'institution du mariage, mais aussi sa brièveté sans divorce, ce qui correspond à l'impératif de diversification des partenaires.

Il reste que tout ceci est étranger aux mœurs de l'ethnie dominante.

30 Cf. Claude LEVI-STRAUSS, La famille, dans : Le regard éloigné, Paris, Plon, 1983, pp. $79,82,84 ; 91,92$. 


\section{B) UN PLURALISME JURIDIQUE CONFLICTUEL}

Il faudra étudier ce en quoi différent les systèmes de parenté en cause.

Nous verrons ensuite les conflits qui en ont résulté.

\section{a) Quels sont les rapports entre les systèmes de parenté Na et Han ? ${ }^{31}$}

Catherine Capdeville fait d'abord remarquer que contrairement à ce que nous pensons trop souvent, filiation et pouvoir ne sont pas forcément liés. Stricto sensu, la filiation désigne uniquement la manière dont une société conçoit l'appartenance des individus à leur groupe primordial de parenté. Les $\mathrm{Na}$ ne sont pas matriarcaux au sens traditionnel de cette expression. Ils sont seulement matrilinéaires. Ce qui signifierait que les femmes n'y ont pas davantage de pouvoirs que dans d'autres sociétés.

Cependant, on peut observer d'après l'exposé des quatre modes de relation au sein de la société $\mathrm{Na}$ tels que je les ai résumés, notamment le plus répandu, celui de la visite furtive, que les femmes $\mathrm{Na}$ sont beaucoup plus libres que les femmes Han : aucun mariage ne leur est imposé, la liberté du consentement est la règle et les parents n'ont que peu de choses à dire (contrairement à l'autorité parentale chez les $\mathrm{Han}$ ) ; les femmes $\mathrm{Na}$ peuvent choisir leurs amants, qui sont nombreux. Autant de traits qui ont toujours suscité l'étonnement, voire la critique, des observateurs étrangers, qu'ils soient chinois, ou occidentaux. Il y a donc bien des différences substantielles entre les $\mathrm{Na}$ et les $\mathrm{Han}$. Ce qui explique d'ailleurs que le gouvernement chinois ait tenté à plusieurs reprises de réformer leurs mœurs. De même, il faut noter que certains Han viennent faire du tourisme sexuel chez les $\mathrm{Na}$, car ils assimilent la visite à de la prostitution, ce qui est évidemment une grave méprise. On peut aussi ajouter que théoriquement, une relation sexuelle entre un père biologique et sa fille est possible, puisqu'il n'y a pas de parenté entre.

Claude Lévi-Strauss avait étudié les Na. Dans un petit article "Le retour de l'oncle maternel ", écrit en 1997, peu après le décès de la princesse Diana, il analysait un discours prononcé aux funérailles de la princesse par son frère, le Comte Spencer. Il disait qu'il voulait assurer son rôle donc maternel vis-à-vis de ses neveux orphelins. Pour Lévi Strauss, il s'agissait de la résurgence d'une ancienne coutume

Catherine Capdeville note en conclusion qu'aucune société ne peut fonctionner sans organiser des rôles différenciés pour les deux sexes, en fonction de leur position dans les différents systèmes de parenté. On peut cependant observer que l'exemple des sociétés occidentales actuelles amène à constater

31 Cf. Catherine CAPDEVILLE, Matriarcat et patriarcat en Chine- Han et Na, Langues Orientales, Inalco 2018, Les femmes à travers le monde, PP.14-19, Hal 02397136. 
une atténuation de cette différenciation des rôles, et même, en droit français, en ce qui concerne la filiation (transmission du nom de famille), la transition d'un système anciennement patriarcal et patrilinéaire à un système de filiation indifférenciée ainsi qu'à une société relativement égalitaire en ce qui concerne les femmes et les hommes.

D'ailleurs, Emmanuel Todd fait remarquer que "le statut de la femme est en réalité plus élevé dans les systèmes de parenté indifférenciée que dans les sociétés matrilinéaires $»^{32}$.

\section{b) Qu'est plus précisément le patriarcat Han?}

Depuis très longtemps, les Han sont organisés en lignages patrilinéaires ${ }^{33}$. Le nom de famille se transmet en ligne directe exclusivement masculine. Le groupe de parenté inclut tous les descendants, y compris les femmes non mariées. Seuls les garçons héritent de leurs parents, les filles reçoivent une dot. On se souvient qu'il en a longtemps été ainsi en France : il a fallu la Révolution française pour que filles et garçons soient traités de façon égalitaire au niveau des successions. L'organisation patrilinéaire n'est pas uniforme dans toute la Chine : elle est moins importante dans le Nord. De plus, dans certaines zones, l'organisation sociale est moins structurée autour de la parenté. Depuis son arrivée au pouvoir, le Parti communiste a cherché à diminuer l'autorité des lignages patrilinéaires. Depuis les années 1980 et la politique de modernisation, les structures traditionnelles sont affaiblies par les transformations économiques, comme cela se produit dans beaucoup d'autres parties du monde.

Ce système patrilinéaire a attiré l'attention de beaucoup d'anthropologues. En 1947, Claude Lévi-Strauss, dans les Structures élémentaires de la parenté s'est servi du système chinois pour former son modèle de l'échange généralisé. Il avait utilisé les travaux du sinologue Marcel Granet ${ }^{34}$.Plus tard, en 1977, Léon Vandermeersch en a parlé dans La voie royale. Les premières enquêtes de terrain ont été effectuées en 1948-1949 par des anthropologues chinois, Francis HSU et Lin YUEH HWA ${ }^{35}$. Pour la fin du siècle précédent, il faut aussi citer le travail d'I.Thireau et HUA LINSHOU ${ }^{36}$.

32 Cf. Emmanuel TODD, L'Origine des systèmes familiaux, Tome I : L'Eurasie, Gallimard, Paris, 2011.

33 Cf. Catherine CAPDEVILLE-ZENG, Un lignage chinois aujourd'hui en Chine du sud est, ENS Paris-Saclay, Terrains et Travaux, 2009 /2, numéro 16, PP.31-53.

34 Marcel GRANET, Les catégories matrimoniales et relations de parenté dans la Chine ancienne, Paris, Felix Alcan,1939.

35 Cf. Y. LIN, The golden Wing, : a sociological Study of chinese Familism, London, International Library of Sociology and Social Reconstruction,1948 ;Francis HSU, Under the Ancestor's shadow, London, Routledge and Keagan Paul, 1949.

36 I.THIREAU et HUA LINSHOU, Enquête sociologique sur la Chine 1911-1949, Etude d'un lignage du Guangdong avant la Révolution, Paris, PUF, 1996. 
Plusieurs auteurs classiques chinois indiquent que dans le passé, les enfants connaissaient leur mère, mais pas leur père ${ }^{37}$. C'était pour eux un état de promiscuité sexuelle. Nous avons vu que cette idée sera reprise par des auteurs occidentaux du XIXe siècle. C'est au moment où les fils ont pu connaître leur père que commence la civilisation. On peut à cet égard rappeler l'origine du terme Patriciat en droit romain. Les patriciens étaient ceux qui pouvaient patrem ciere, c'est-à-dire citer le nom de leur père, par opposition à la plebs, supposée confuse. La patrilinéarité serait donc un progrès. Cependant, on peut noter que la Chine fait preuve d'un respect fondamental pour les mères et croit à la nécessité de la coexistence des sexes, donc du mariage, conçu presque uniquement à des fins procréatives.

De plus, certains travaux récents incitent à plus de réserve sur l'existence d'un véritable patriarcat, dans le sens d'un pouvoir exclusif des hommes. En 2003, Nicolas Zafferey explique que la vision de la femme chinoise conçue comme l'inverse de la femme occidentale supposée libre est une invention des réformateurs du début du XXIe siècle, qui a servi de base à la volonté du Parti communiste d'éradication des coutumes ${ }^{38}$. En 2009, l'anthropologue Charles Stafford, décrit le pouvoir réel des femmes chinoises ${ }^{39}$.

D’une manière générale, dans la Chine traditionnelle, le célibat était considéré comme suspect ${ }^{40}$.Mais sous la dynastie Zhou (10 45 av. J.-C.-481 av. J.-C.) une grande liberté sexuelle régnait dans les classes populaires. Le taoïsme soulignait l'importance de la complémentarité entre les sexes et vénérait le sexe féminin. Mais le confucianisme dans sa définition de l'harmonie subordonnait le sexe féminin au sexe masculin, ce qui entraînera la formation du patriarcat.

D'autre part, quels sont les sentiments caractérisant les différents types d'union ? L'anthropologue chinois Xiaotong FEI, dans son enquête sur son pays natal de la province du Jiangxu, en $1947^{41}$, écrit que la société rurale chinoise n'est pas orientée par l'amour, mais que règne plutôt une certaine distance, une réserve, entre gens mariés. Ils sont ainsi protégés des dérives passionnelles, qui pourraient être une menace pour l'ordre social. Cependant, d'autres sources, notamment romanesques et poétiques, montrent que la Chine connaissait des formes individuelles d'amour, tout en les situant en dessous des relations

37 Cf. Catherine CAPDEVILLE, Matriarcat et patriarcat en Chine- Han et Na, Langues Orientales, Inalco 2018, Les femmes à travers le monde, PP.14-19, Hal 02397136.

38 Cf. Nicolas ZAFFEREY, La condition traditionnelle de la femme en Chine-Etat de la recherche, Etudes Chinoises, Volume 22, 2003.

39 Dans : Suzanne BRANDSTÄTER-Gonzalo SANTOS, Chinese Kinship, Contemporary Anthropological Perspectives, 2009, 137-153.

40 Cf. Catherine CAPDEVILLE-ZENG, Mariage et amour en Chine-Les échanges et les sentiments dans les mariages dans un village du Jiangxi d'aujourd'hui,, Quatriéme Congrés du Réseau Asie et Pacifique, Septembre 2011, Paris, HA 01282547.

41 Cf. Xiaotong FEI, From the Soil, The foundations of Chines Society, University of California Press, Berkeley, 1947. 
familiales. L'amour romanesque se pratiquait avec les courtisanes, qui, dans les classes aisées, pouvaient devenir des concubines. La famille était ainsi protégée contre les divorces.

Mais au début du XXe siècle, les jeunes intellectuels chinois urbains se déclarent en faveur du consensualisme des époux, précédant ainsi les communistes, qui, en 1950, promulguent une loi interdisant le mariage arrangé et le concubinage. Mais dans la pratique, les vieilles coutumes vont subsister pendant longtemps. Après le chaos de la Révolution culturelle qui voit la multiplication de divorces pour des causes politiques (comme sous la Révolution française), les aspirations individualistes ressurgissent.

Elles s'accentuent avec la modernisation économique des années 80. Des enquêtes sur le terrain montrent le déclin du choix du conjoint par les parents. Le mariage n'est plus simplement un instrument de procréation, il doit être le cadre de sentiments personnels et de la recherche du bonheur individuel. En 1981, Hu Yaobang, secrétaire général du parti communiste chinois, pourtant un libéral, dit:

"Le Parti n'est pas opposé à l'amour. Mais il est important de se rappeler qu'il y a d'autres choses que l'amour ". De façon ultime, l'amour relève donc moins des individus que de l'Etat.

Rappelons que dans l'histoire du droit occidental, il y eut une lutte pluriséculaire entre le consensualisme, prôné par l'Eglise, et les coutumes, relayées par le droit royal, qui faisait du consentement des familles, c'est-à-dire des pères de famille, la condition du mariage.

Dans tout ce qui précède, on voit donc que ce qu'on appelle le patriarcat chinois, même si le système han est incontestablement patrilinéaire, doit être apprécié avec nuance. L'amour individuel, l'attraction sexuelle, l'amour romanesque sont communs au Han et aux $\mathrm{Na}$. Mais leur structure familiale diffère largement. De plus, et peut-être surtout, en raison des théories évolutionnistes, les coutumes $\mathrm{Na}$ ont été considérées comme les vestiges d'un état arriéré qu'il fallait donc réformer dans le sens du progrès. On trouve cette attitude chez bien d'autres colonisateurs en Occident ${ }^{42}$. En tout cas, ce présupposé explique que le pouvoir central chinois, depuis l'arrivée au pouvoir du Parti communiste, va tenter plusieurs réformes des coutumes $\mathrm{Na}$. Il n'y réussit globalement pas.

C'est en ce sens qu'on peut parler, aujourd'hui encore, d'un pluralisme juridique en Chine ( cf. Norbert ROULAND, Some reflexions about legal pluralism, in: Life World and Plural Norms from the Perspective of Legal

42 Cf. Norbert ROULAND, Les colonisations juridiques : de l'Arctique à l'Afrique Noire, Journal of legal pluralism and unofficial law, 23 /1990 ,39-162. En ce qui concerne la Russie, cf. Anatoly KOVLER, Les peuples autochtones de Russie :le droit à un statut juridique différent, dans : Norbert ROULAND(dir.), Le droit à la différence, Aix en Provence,PUAM, 2002, 127135. 
Anthropology, organised by Pr. Wang Weichen, Lecture in Shanghai Normal University, September 2020, (en chinois et en anglais).

\section{c) Le pouvoir central chinois et le droit coutumier $\mathrm{Na}^{43}$}

Il est intéressant de citer tout d'abord des allocutions du Président XI JI PING (toutes les citations sont extraites de : XI JI PING, La Gouvernance de la Chine, Editions en langues étrangères, Beijing, 2014). On procédera ensuite à un bref rappel historique.

\section{1) LA POLITIQUE ACTUELLE:LES DISCOURS DU PRÉSIDENT XI JI PING}

Les civilisations se diversifient et s'enrichissent à travers les échanges et l'inspiration mutuelle qui constituent un moteur important pour le progrès de l'humanité, la paix et le développement dans le monde. Les civilisations sont diverses et cette diversité donne un sens à leurs échanges et à leur inspiration mutuelle [...] Tout comme la lumière solaire qui compte sept couleurs, notre monde est multicolore [...] Les civilisations sont égales, et cette égalité rend possibles leurs échanges et leur inspiration mutuelle. Les différentes civilisations de l'humanité sont égales en valeur, et chaque civilisation a ses atouts et ses faiblesses. Il n'existe pas de civilisation parfaite dans le monde, ni de civilisation inutile. Aucune civilisation n'est supérieure ou inférieure aux autres [...] l'histoire nous enseigne que seuls les échanges et l'inspiration mutuelle assurent la pleine vitalité d'une civilisation (Intervention au Sommet sur la sécurité nucléaire tenue à La Haye,aux Pays-Bas, 24 mars 2014).

C'est seulement lorsque l'on assure l'égalité de tous les citoyens devant la loi, que l'on respecte et protège les droits de l'homme, et que l'on assure en vertu de la loi les droits et la liberté du peuple, que la Constitution peut profondément pénétrer le cour du peuple et que celui-ci l'applique consciencieusement dans toutes ses actions. [...] Nous devons approfondir la réforme de l'appareil judiciaire et veiller à ce que le pouvoir judiciaire et le pouvoir de contrôle soient exercés en vertu de la loi et de manière indépendante et équitable.

(Discours prononcé le 4 décembre 2012 lors de la réunion des différents milieux sociaux de la capitale à l'occasion de la commémoration du 30 anniversaire de la promulgation de la Constitution en vigueur).

Depuis trente ans, grâce à sa position suprême et sa puissante force légale, la Constitution chinoise garantit le statut des citoyens en tant que maîtres du pays, favorise la réforme, l'ouverture et la modernisation socialiste, fait avancer le processus pour construire un État de droit socialiste, promeut le développement des droits de l'homme, et sauvegarde de l'unité nationale,

43 Cf. CAI HUA, op.cit., 289-309. 
la solidarité interethnique et la stabilité sociale [...]la nation chinoise multiethnique, tous les organes de l'État, toutes les forces armées, tous les partis politiques, tous les mouvements sociaux, toutes les entreprises et tous les établissements d'intérêt public doivent prendre la Constitution comme critère fondamental de leurs activités, et sont chargés de sauvegarder la majesté de la Constitution et d'assurer son application. Il est interdit à toute organisation ou tout individu d'avoir le privilège d'être au-dessus de la Constitution et des lois.

(Discours prononcé le 4 décembre 2012 lors de la réunion des différents milieux sociaux de la capitale à l'occasion de la commémoration du $30^{\mathrm{e}}$ anniversaire de la promulgation de la Constitution en vigueur).

La lutte anti-terroriste est liée à la sécurité nationale, aux intérêts vitaux du peuple, à la situation générale de la réforme, du développement et de la stabilité. Il s'agit d'une lutte destinée à sauvegarder l'unité de la patrie, la stabilité de la société et le bonheur du peuple.

Les terroristes violents ignorent les droits fondamentaux de l'homme, piétinent l'humanisme et la justice et défient le seuil de tolérance de la civilisation bumaine. Ce n'est ni une question d'ethnie, ni une question de religion, mais l'ennemi commun de notre peuple multi-ethnique [...] nous devons intensifier la lutte contre le séparatisme, et en portant haut levé le drapeau de la grande union de toutes les ethnies, insister sur la promotion de la solidarité multi-ethnique et de la prospérité commune. Nous devons mener en profondeur la sensibilisation du public à la solidarité interethnique, consolider le fondement idéologique en la matière et réunir autant que possible les peuples de toutes les ethnies. [...]Il faut faire progresser énergiquement l'État de droit afin de mieux protéger les intérêts légitimes du peuple et encourager celui-ci à résoudre les divers conflits par des procédures juridiques et des moyens légaux. (Sauvegarder la sécurité nationale et la stabilité sociale, propos tenus le 25 avril 2014 lors de la $14^{\mathrm{e}}$ séance d'études du Bureau politique du $18^{\mathrm{e}}$ Comité central du Parti).

La place me manque pour organiser pour analyser en détails tous ces discours. J'en retiendrai seulement l'affirmation de certains principes importants par rapport au sujet de cette communication : l'égalité entre toutes les cultures (aucune n'est inférieure ou supérieure à une autre), la nécessité des échanges interculturels ; l'égalité de tous les citoyens chinois devant la loi ; le caractère multi-ethnique du peuple chinois ; la nécessité d'assurer la protection des droits de l'homme et leur développement ; la nécessité de poursuivre la construction de l'État de droit socialiste. ; la résolution des conflits par des procédures juridiques.

Comment cela se traduit-il plus précisément au niveau juridique en ce qui concerne les minorités nationales chinoises ? ${ }^{44}$

44 Pour un point de vue critique, cf. Mélane RODRIGUES, Les régions autonomes chinoises : vitrines du multiculturalisme, Rapport de recherche IEP Aix en Provence, M2 Expertise internationale,2019/2020. 


\section{2) BREVE HISTOIRE DU STATUT JURIDIQUE DES MINORITÉS NATIONALES ${ }^{45}$}

Quelle était la condition des peuples allogènes dans la tradition impériale chinoise? ${ }^{46}$

Pour les Chinois, la civilisation reposait sur la sédentarisation, la construction de villes, la pratique de l'agriculture, le mariage monogamique et la capacité à respecter un ordre politique régi par des lois. Autant de traits qui ne correspondaient pas à ceux des peuples allogènes. La racine "chien " se retrouve dans beaucoup d'idéogrammes désignant ces peuples. Si les Barbares faisaient l'effort d'accepter les mœurs des Han, ils pouvaient trouver place dans l'Empire.

La première période va de 221 avant notre ère jusqu'en 1253. Quand les chefs indigènes (tusi) se soumettaient, le gouvernement central les laissait libres de continuer à régir les populations comme ils le souhaitaient, sans modifier leur organisation sociale. Les chefs gardaient leur titre de façon héréditaire. Sous la dynastie des Tang (618-907 après J.-C), l'empereur Li Shimin affirme : " Depuis les temps anciens, tout le monde prend les Han comme supérieurs et les ethnies minoritaires comme inférieures. Moi, je les aime tous comme identiques".

Au cours de la seconde période (1253 -1368) le gouvernement central envoie des mandarins pour exercer le pouvoir conjointement avec les chefs indigènes. Par la suite, considérant que les Mongols avaient eu trop souvent recours à la force vis-à-vis des ethnies minoritaires, la dynastie des Ming (13681644 ) a mis en œuvre des politiques visant à gagner la sympathie des ethnies minoritaires et à les soumettre pacifiquement. De 1644à 1911, la dynastie des Qing, fondée par une ethnie minoritaire, les mandchous, adopte des politiques beaucoup plus sévères. Elles visent à minimiser les pouvoirs des chefs indigènes au profit de ceux de magistrats nommés par le pouvoir central. C'est de cette dynastie que date véritablement l'influence du gouvernement central, marquée notamment par l'instauration de l'impôt.

L'empereur Wudi (140-87) de la dynastie Han, seconde dynastie impériale chinoise, lance une politique de conquêtes. Les peuples soumis gardent leurs chefferies et leurs coutumes, moyennant tribut et l'installation de garnisons chinoises. Il faut cependant noter, sur le plan historique, une tendance à l'affirmation de la supériorité des Han. Jusqu'à la dynastie mandchoue, on distingue en ce qui concerne les ethnies périphériques entre les barbares crus, ceux qui gardent leurs traditions et les barbares cuits, ceux qui sont acculturés.

45 Cf. Vanessa FRANGVILLE, L'unité dans la diversité : l'altérité en Chine, moteur de l'unité nationale, Univ. Jean Moulin Lyon3, Discours sur l'autre, Discours sur soi : Constuctions identitaires face à l'altérité, Univ. Jean Moulin Lyon 3, pp. 37-57,2007, Halsshs -00308295.

46 Cf. les développements de Jacques POUMAREDE, dans N.ROULAND ,op.cit.(Droit des minorités et des peuples autochtones), 135 sq. 
Wang FUZHI, philosophe chinois, écrit au XVIIème siècle :

"Il y a sous le Ciel, deux grandes lignes de défense: l'une, sépare les Chinois des Barbares; l'autre, les gens de bien (junzi) des gens de rien (xiaoren $[\ldots]$; il ne convient pas que le désordre s'installe entre (eux et nous). Quand il s'y installe, les principes suprêmes de l'humanité sont détruits, et les populations de la Chine, victimes de la voracité de ces gens, sont plongées dans la douleur et l'affliction.".

Dans la Chine du Sud, les Han ont été en contact avec des peuples autochtones qu'ils ont au contraire voulu assimiler. Ils ont pratiqué une politique d'administration indirecte en nommant des administrateurs appartenant aux notables autochtones. Les élites étaient fortement incitées à se convertir aux mœurs des Han. Une politique qui rappelle celle de la promotion des élites provinciales, qui fut un des ciments de l'Empire romain, qui, par ailleurs, laissait intactes les coutumes et les religions des peuples qu'il conquerrait. En Egypte le pouvoir romain laissa longtemps subsister des mariages entre frères et sœurs contraires au droit romain (pratiqués par environ vingt pour cent des Egyptiens).

$\mathrm{Au}$ XIXe siècle, les puissances européennes s'efforcèrent de maintenir la Chine dans un état de faiblesse. C'est pourquoi elles soutinrent les menées séparatistes à la périphérie de l'empire : les Russes sur la frontière de l'Amour, les Anglais au Tibet, les Français dans le Yunnan. Les Japonais, pour leur part, poussaient à l'autonomie de la Mandchourie. Dans ces conditions, il est logique que la I ere République chinoise ait été fondée sur la base d'un État unitaire composé de citoyens chinois égaux en droit, excluant la reconnaissance des droits des minorités. Un nationalisme de type jacobin a caractérisé l'idéologie du Guomindang.

Comment la situation a-t-elle évolué dans la RPC ?

Après la prise du pouvoir par les communistes commence la célébration de la diversité culturelle chinoise.

Le préambule de la Constitution chinoise de 1982 affirme:

"La Chine est l'un des plus anciens pays du monde. Ses diverses nationalités, qui ont toutes contribué à créer une brillante culture, possèdent de glorieuses traditions révolutionnaires».

Les régions autonomes possèdent le droit de s'administrer librement dans un certain nombre de domaines. Les ethnies gèrent leurs affaires intérieures (article 30 de la Constitution de 1982). Cette autonomie se traduit sur le plan politique par la mise en place d'institutions autonomes, consacrée en 1984 par la loi sur l'autonomie régionale des minorités nationales. Sont instituées des assemblées populaires élues au suffrage indirect qui représentent les minorités nationales et servent d'intermédiaire avec le pouvoir central. Les parlements locaux peuvent voter des lois autonomes et travailler dans leurs langues nationales. 
En 1953 est lancée la première grande campagne de recensement de l'Histoire du pays. Au total, 55 nationalités sont officiellement identifiées et éligibles au statut de "minorité nationale ". Cette identification s'accompagne de l'octroi de droits spécifiques pour chacune de ces minorités et d'une représentation au Congrès. A cela s'ajoute la possibilité de se proclamer en tant que région autonome si le poids démographique de l'ethnie au sein du territoire est supérieur à $22 \%$. Ce revirement politique à l'égard des minorités est consacré en 1959, date qui marque le 10ème anniversaire de la RPC. Pour la première fois dans l'Histoire du pays, les minorités sont invitées à venir célébrer la fête nationale. Symboliquement, cet événement confirme leur intégration au sein de la nation.

Cette reconnaissance se traduit par un refus de l'ethnocentrisme Han. Férocement combattu par MAO ZEDONG, ce " chauvinisme Han » apparait dans le Préambule de la Constitution qui affirme "il faut combattre le nationalisme de grande nationalité - surtout le chauvinisme Han [...] ». Lors de la réforme constitutionnelle de 1975 qui donne naissance à une nouvelle Constitution, l'article 4 affirme "les nationalités sont égales en droit.».

Pour comprendre ce revirement politique il convient de revenir sur un concept essentiel développé par Staline : celui de "nationalité. " Ainsi, il définit en 1913 la nation comme "une communauté de populations stables, historiquement constituées, ayant une langue, un territoire, une vie économique et une culture commune. " La prise en considération de cette définition par le PCC ne doit pas être ignorée. Bien qu'assouplie, elle est à la base de la politique menée durant cette période. L'extrait d'un article paru dans le journal Le Monde en 1954 confirme cette thèse. Pendant un court entretien avec le président de la Mongolie-Intérieure de l'époque, ce dernier présente l'autonomie accordée aux minorités comme un moyen "d'élever " l'économie et la culture des régions alors présentées comme retardataires afin d'équilibrer le niveau de vie entre les différents territoires.

Mais la compréhension stalinienne du concept de nationalité va plus loin. En effet, Staline propose également une hiérarchisation des différentes nationalités selon leur stade d'évolution. On retrouve ici la différenciation entre Han et non-Han, et un rapport plus général aux théories évolutionnistes, notamment marxistes, citées plus haut.

L'arrivée au pouvoir de Deng XIAOPING en 1977 marque le retour à la politique d'assimilation et un accroissement de la pauvreté des minorités au sein des régions autonomes. Entre 1957 et 1990, la proportion d'ethnies minoritaires membres du Parti stagne, passant de $5.5 \%$ à $5.7 \%$. La situation de faible développement économique vient également ternir le bilan. Un article 
paru en $1999^{47}$ affirme que les minorités représentent $43,3 \%$ de la population en situation d'extrême pauvreté.

Au début du XXI siècle, le gouvernement central réalise chez les minorités des infrastructures routières, électrifie les villages, améliore les rendements agricoles $^{48}$.

C'est à la lumière de ces évolutions et de leurs alternatives qu'il faut maintenant étudier les politiques de réforme des mœurs matrimoniales des $\mathrm{Na}$ par les gouvernements chinois : un cas de pluralisme juridique.

\section{3) L'ÉCHEC DES TENTATIVES DE RÉFORME DU DROIT COU- TUMIER DES $N A^{49}$}

Le Pr Cai Hua leur consacre tout un chapitre, que je vais résumer.

En 1950 a été fondé dans le district du Ninglang un « Gouvernement démocratique des multiples ethnies unies "; ses principaux responsables étaient des Han. Ils ont jugé que la visite furtive était une " coutume arriérée et primitive ". Elle était immorale et contraire à la législation matrimoniale chinoise. Ils avançaient les arguments suivants : plus de $50 \%$ des adultes étaient atteints de diverses maladies sexuelles; une bonne partie des femmes était stérile ; elle perturbait la production dans la mesure où les hommes ne pensaient qu'à faire des visites.

La première mesure date de 1956, année de la réforme agraire. Afin de porter atteinte au système matrilinéaire, un règlement est adopté suivant lequel la distribution de la terre s'effectuera en fonction de l'habitation des hommes. Si un homme veut fonder un foyer tout seul, la terre lui sera distribuée, au lieu d'être distribué à sa famille maternelle. Cette mesure fut totalement inefficace, aucun $\mathrm{Na}$ n'ayant voulu en bénéficier, au grand étonnement du gouvernement local.

En 1958, lors du Grand Bond en avant, le gouvernement local se prononce en faveur de la supériorité de la monogamie socialiste par rapport aux visites furtives. Il tente d'officialiser les visites furtives de longue durée. Cela les transformerait en un mariage monogame de fait. Les jeunes sont incités à se marier entre ou avec des cadres d'autres ethnies. Ceci n'a rien donné. Les Han n'arrivaient pas à comprendre qu'un conjoint, mari ou épouse, puisse être considéré comme un étranger par rapport à son partenaire. La politique gouvernementale reçoit l'appui des ethnologues qui ont publié les rapports d'enquête en 1960 et 1963 : il faut réformer ce système arriéré.

47 Cf.N. BECQUELIN (1999), Tensions interethniques et pauvreté endémique, Perspectives Chinoises 39, pp.19-28.

48 Cf. Françoise GRENOT-WRANG, La Chine du Sud-La mosaïque des minorités, Paris, Les Indes Savantes,2017,13.

49 Cf. CAI HUA, op.cit., 289-309. 
En 1966 et 1971, on va de nouveau tenter d'imposer la monogamie.

En 1966, au début de la Révolution culturelle, Mao Dze Dong incite les Chinois à balayer les "Quatre vieilleries": anciennes coutumes, anciennes habitudes, ancienne moralité et ancienne culture. Certains couples se forment, mais ils se dissolvent rapidement.

En 1974, le gauchisme est à son paroxysme. Une autre tentative de réforme matrimoniale va avoir lieu. Le gouverneur provincial du Yunnan fait une inspection personnelle sur le terrain et déclare :

«Il faut réformer avec résolution le système matrimonial arriéré des Naxi .Réformer cet ancien système matrimonial relève du cadre de la lutte des classes dans le domaine idéologique et constitue donc une révolution dans le domaine de la superstructure».

Les anciens arguments sont avancés : la visite furtive affecte les activités de production; elle est nuisible à la solidarité entre mari et épouse en favorisant les adultères; elle est préjudiciable à l'éducation des enfants ; elle porte préjudice à la formation de moralité et de qualités communistes dans la masse.

Les mesures éducatives ne suffisent pas; il faut passer à la contrainte. Tous ceux qui ont moins de 50 ans et vivent en situation de visite furtive de longue durée doivent se marier. Tout enfant naturel ne pourra obtenir sa ration annuelle de céréales que lorsque sa mère aura désigné avec certitude qui est son géniteur et celui-ci devra fournir à son enfant ses moyens de subsistance jusqu'à ce qu'il ait 18 ans. Après le mariage, la résidence commune est obligatoire. Comme les autorités avaient observé que lors des précédentes tentatives de réformes, cellesci étaient bloquée par les femmes âgées, elles vont être particulièrement mises au courant de la nouvelle réforme.

Du coup, les villageois ont peur de faire des visites furtives, craignant par la suite d'être obligés de se marier. Le Pr. Cai Hua cite des témoignages :

"Une épouse et un mari (monogamie) est la qualité et la caractéristique des Han; nous, les Na, ne vivons pas de cette manière. Rendre visite aux femmes sans se mettre ensemble s par couple est notre caractéristique. Si tous ensemble nous devons pratiquer la monogamie, nous deviendrons des Han et les Na disparaîtront».

On ne saurait mieux dire....

Autre témoignage :

«Pour le moment, nous nous sommes mis ensemble, mais ce n'est que pour appliquer la politique du gouvernement. Nous espérons pouvoir retourner chacun à notre maison natale, plus tard, lorsque la politique nous le permettra. Pourvu que la politique nous laisse vivre en note de visite...»

Concrètement, le désordre règne dans tous les villages. Certains couples se dissolvent. D'autres sont relativement stables : soit ceux qui ont fondé une famille nucléaire, soit ceux dont la lignée d'accueil était peu nombreuse. De 
juin à août1976, quatre chercheurs de l'Institut de l'histoire du Yunnan se rendent sur le terrain. Ils constatent des résistances. Ils concluent qu'il faut accentuer les efforts de réforme. Ils n'arrivent pas à s'expliquer comment ce système matrilinéaire arriéré a pu se maintenir alors que la Chine d'aujourd'hui se retrouve au stade socialiste. Au total, plus de la moitié des mariages plus ou moins forcés se termineront par une séparation.

A la fin du siècle précédent, même si faire des enfants hors mariage est toujours considéré comme_immoral et illicite, la politique s'assouplit à partir de 1981. En 1982 les lignées obtiennent que chacune ait sa propre terre. Mais des amendes pénalisent la naissance des enfants naturels. Les villageois ne dépendent du gouvernement local que pour peu de choses. Ils essaient de plus en plus de détourner les règles administratives. Par exemple, un villageois déclare qu'il est le géniteur des enfants de plusieurs femmes de différentes lignées à chaque fois qu'une lignée risque d'être frappée par l'amende. En effet, quand quelqu'un reconnaît être le géniteur de l'enfant, l'amende est levée. C'est donc un service que certains individus rendent aux femmes.

Mais il existe d'autres facteurs que les mesures coercitives.

Les instituteurs sont maintenant des $\mathrm{Na}$, mais ils appliquent la politique gouvernementale.

Cela d'autant plus que tous les manuels étant rédigés en chinois par des $\mathrm{Han}$ (le $\mathrm{Na}$ est une langue sans écriture), les textes sont imprégnés des valeurs Han. Par exemple, ils enseignent que tous les enfants doivent connaître leur père.

Les cadres $\mathrm{Na}$ qui travaillent dans les différents organismes du Parti communiste des gouvernements sont payés par l'État et il leur est interdit de vivre en mode de visite. Si cela se produit, le cadre perd son emploi. Ces cadres pratiquent souvent une politique de compromis. Ils expliquent que la visite furtive était une modalité exceptionnelle ; et dans la pratique, ils ne s'opposent pas aux comportements déviants par rapport à la législation matrimoniale nationale. Chez les jeunes, on note à la fois la pratique de la visite furtive et de la relation ouverte.

À l'époque de Deng Xiaoping, un garçon de 29 ans dialogue avec le Pr. Cai Hua:

«As -tu une amie ouverte?

- Oui.

- Mais si tu aimes surtout t'amuser avec des filles, pourquoi alors faire la visite ouverte?

- Tu sais, la politique peut changer. Si je fais la visite ouverte avec une fille, c'est que je n'ai pas envie qu'un jour la politique change de nouveau, et qu'on m'impose alors n'importe quelle fille simplement parce que j'ai couché un certain nombre de fois avec elle. Si on me demande à ce moment de me marier, 
il vaut mieux que je puisse le faire avec une fille que j'aime quand même un peu plus que les autres".

A la fin du siècle précédent, même si faire des enfants hors mariage est toujours considéré comme_immoral et illicite, la politique s'assouplit à partir de 1981. En 1982 les lignées obtiennent que chacune ait sa propre terre. Mais des amendes pénalisent la naissance des enfants naturels. Les villageois ne dépendent du gouvernement local que pour peu de choses. Ils essaient de plus en plus de détourner les règles administratives. Par exemple, un villageois déclare qu'il est le géniteur des enfants de plusieurs femmes de différentes lignées à chaque fois qu'une lignée risque d'être frappée par l'amende. En effet, quand quelqu'un reconnaît être le géniteur de l'enfant, l'amende est levée. C'est donc un service que certains individus rendent aux femmes.

Tout ceci témoigne d'un profond malentendu culturel. Les réformateurs et les villageois parlent de "famille» et «solidarité», mais ces termes ne recouvrent pas les mêmes concepts. Pour les réformateurs, la famille, c'est le mari, l'épouse et les enfants. Pour les $\mathrm{Na}$, ce sont les frères et des sœurs et les enfants de ces dernières de chaque génération. C'est la cause la plus profonde de l'échec des réformes. On peut y ajouter que le mariage recommandé par les réformes est en fait un mariage plus ou moins contraint. Alors que justement la législation matrimoniale chinoise interdit les mariages arrangés.

Sur le plan théorique, l'échec de ces différentes réformes montre que malgré la pression des représentants des autorités centrales, les $\mathrm{Na}$ ont réussi à vivre en situation de pluralisme juridique.

Est-ce que l'attitude du gouvernement central chinois est exceptionnelle? Absolument pas.

\section{C) LA POLITIQUE DES GOUVERNEMENTS CHINOIS ET LES DONNÉES COMPARATIVES}

Je citerai d'abord deux exemples asiatiques avant de pousser plus loin la comparaison.

\section{a) Deux exemples asiatiques extérieurs au monde chinois}

Je prendrai d'abord un exemple indonésien, puis un exemple indien.

\section{1) UN PLURALISME JURIDIQUE MAINTENU : LES MINANGKA- $B A U$ D'INDONÉSIE}

En Indonésie ${ }^{50}$, les Minangkabau de Sumatra étaient matriarcaux. Ils sont aujourd'hui encore le peuple matriarcal le plus connu. Mais ils subissent l'influence de systèmes patriarcaux auxquels ils opposent une vive résistance. Ils

50 Cf. Heide GOETTNER-ABENDROTH, op.cit., 209 sq. 
obéissent à la matrilinéarité, la matrilocalité, et les hommes sont des visiteurs qui vont et viennent entre la maison de leur mère, où ils résident, et celle de leur épouse, où ils ne sont que des invités. Au XIVe siècle, le roi javanais minangkabau a essayé d'imposer la patrilinéarité en prenant l'exemple patriarcal hindou. Ceci a échoué. Deux siècles plus tard, l'islamisation se produit à Sumatra, mais l'islam patriarcal n'est accepté qu'en apparence. Dans la seconde partie du XXe siècle, le gouvernement central indonésien essaye de réduire l'autonomie des femmes minangkabau. De plus, un certain nombre d'hommes quitte les villages pour aller travailler dans les villes. Mais les terres appartiennent aux épouses, ce qui les protège en cas de divorce. En outre, en matière d'héritage, la propriété privée qu'un homme aurait pu acquérir ne peut être détenue que pendant une génération, en tant que don fait à ses enfants. Ensuite, elle revient au clan de sa mère.

\section{2) LE TRIOMPHE DU DROIT COLONIAL: LES NAYAR D'INDE DU SUD ET LA COLONISATION BRITANNIQUE.}

En Inde ${ }^{51}$, les cultures matriarcales de l'Inde du nord-est sont similaires à celles des peuples du Tibet et de l'Asie de l'Est. L'Inde du nord-ouest a été influencée par les cultures matriarcales de l'Asie de l'Ouest. Celles-ci se sont maintenues longtemps jusqu'à l'arrivée d'envahisseurs patriarcaux venant également du nord-ouest.

L'Inde du Sud a connu également des sociétés matriarcales, notamment celles des Nayar et des Kashi. Dans la vie traditionnelle Nayar, les femmes gèrent les maisons claniques et chaque mère clanique est la maîtresse de sa maison clanique. La société Nayar est matrilinéaire et matrilocale : les filles et les fils continuent à vivre dans la maison de la mère. Les affaires intérieures du clan sont gérées par la femme la plus âgée, la mère clanique. L'homme le plus âgé n'a aucune autorité sur la lignée. La mère clanique et le frère de la mère sont sur un pied d'égalité et partagent la gestion quotidienne des affaires du clan. L'oncle maternel exerce l'autorité sur le fils de ses sœurs.

Les Nayar se sont principalement installés sur la côte de Malabar. Il y a deux mille ans, les brahmanes védiques atteignent cette région. Il faut souligner une contradiction entre le système des castes hindouiste et le matriarcat, dans la mesure où ce système des castes repose sur une hiérarchie qui n'existe pas dans les sociétés matriarcales. Pour les brahmanes, les Nayar sont "impurs ». Certaines fonctions du corps des femmes, vénérées dans les sociétés matriarcales, sont considérées comme impures, comme dans d'autres religions patriarcales : la menstruation, la grossesse, l'accouchement. Les hommes brahmanes sont les personnes les plus pures et les plus parfaites. Le mariage est indissoluble, il est

51 Cf. Heide GOETTNER-ABENDROTH, op.cit., 389 sq. 
interdit de se remarier. Les veuves doivent monter sur le bûcher après la mort de leur mari, une coutume que les Britanniques vont interdire. Une fille ne peut se marier qu'avec un homme de castes supérieures, ce qui accentue le système patriarcal : l'épouse sera toujours en-dessous de son mari. La naissance des filles, comme dans la société patriarcale chinoise, est sous-évaluée. Un proverbe hindou affirme : "Celui qui a une fille laboure le champ du voisin". En effet, dans le système patriarcal, la fille quitte sa famille d'origine.

Mais dans cette région, les brahmanes ont été dès les débuts dépendants du soutien des Nayar, un peuple guerrier, si bien qu'ils ont dû conclure un compromis historique. Les Nayar ont adopté le système des castes ; mais ils sont devenus la seconde caste après les brahmanes et ont obtenu de leur part une reconnaissance officielle, qui leur a permis de conserver leurs modes de vie matriarcaux.

En revanche, la colonisation anglaise, au début du XIXe siècle, a gravement atteint les formes matriarcales subsistantes ${ }^{52}$. Vers le milieu du siècle, se forme un groupe de jeunes migrants Nayar urbains qui, de fait, deviennent les collaborateurs du pouvoir britannique. Ils ont été éduqués dans des collèges britanniques et ont rapidement occupé la majorité des postes administratifs d'État et exercent des professions de la classe moyenne : enseignants, avocats et médecins. Ils sont également devenus chrétiens. Dès lors, ils étaient convaincus que le système matriarcal était une coutume arriérée qu'il fallait faire disparaître pour accéder au progrès et à la prospérité. En 1912 la polyandrie devient une infraction pénale et est remplacée par la cérémonie de mariage hindou monogamique. En 1913, la famille matriarcale est abolie par la loi. En 1925, la division des propriétés foncières claniques est autorisée, ce qui entraîne leur démantèlement et leur morcellement en propriétés privées. Après l'indépendance, en 1956, une loi abolit le droit d'héritage en ligne féminine et déclare l'héritage possible uniquement en ligne masculine. La chasteté avant le mariage pour les femmes, leur fidélité inconditionnelle dans le mariage, l'autorité du père biologique sur les enfants deviennent les valeurs de l'Inde supposée moderne. Il en résulte que l'autonomie des femmes Nayar a été fortement dégradée. Ceci dit, dans la pratique, la plupart des foyers Nayar sont fondés sur une combinaison d'héritages masculin et féminin, et, en fonction de qui possède le plus de biens, sont tantôt virilocaux (résidence dans la maison du mari), tantôt matrilocaux (résidence dans la maison de la femme) .

D'autres peuples, du nord-ouest de l'Inde, comme les Sinté et les Rom, ont eu plus de chance. Ils affichent encore aujourd'hui des traits matriarcaux prononcés. Les femmes jouissent d'une haute considération et de la liberté

52 La colonisation britannique a également dévalué la situation des femmes artistes hindoues : cf. Norbert ROULAND, A la découverte des femmes artistes-Une histoire de genre, Aix en Provence, PUAM, 2016,69sq. 
sexuelle ; elles exercent les mêmes métiers que les hommes ; elles dominent les clans matrilinéaires. Mais les enquêtes sur le terrain chez eux restent difficiles : ils se méfient des étrangers et des ethnologues et restent donc très discrets sur leurs coutumes.

\section{b) Les colonisations occidentales :la lutte contre le pluralisme juridique au nom des droits de l'Homme.}

Quand J. Ferry, homme de gauche, proclame le droit des races supérieures de coloniser les inférieures: "Il y a pour les races supérieures un droit, parce qu'il y a un devoir pour elles. Elles ont le devoir de civiliser les races inférieures ". le député J. Maigne lui rétorque: "Vous osez dire cela dans le pays où ont été proclamés les droits de l'Homme !». À quoi J. Ferry répond : “... si la Déclaration des droits de l'Homme a été écrite par les Noirs de l'Afrique équatoriale, alors de quel droit allez-vous leur imposer les échanges, le trafic? Ils ne vous appellent pas" ${ }^{33}$. Il avouait ainsi qu'outre-mer, les droits de l'Homme devaient céder aux exigences économiques.

En Afrique noire ${ }^{54}$, presque tous les Etats coloniaux ont garanti le respect des usages et des coutumes propres aux peuples colonisés, tout en leur imposant des droits européens dans un certain nombre de cas. En général, le droit familial fut peu touché. Il comportait cependant certains traits considérés comme immoraux : lévirat, sororat, polygamie, mariage-fantôme, facilité du divorce, etc. Une attitude de prudence : on ne voulait pas provoquer des mouvements de révolte chez les colonisés.

Les Français se lancent dans des entreprises de rédaction des coutumes. En 1905, le gouverneur Roume prescrit aux juges de rassembler les renseignements qui serviront de base à la rédaction d'un coutumier général. Mais il ne s'agit pas seulement de rédiger les coutumes, mais aussi de les transformer. Ses propos sont parfaitement explicites :

"Notre ferme intention de respecter les coutumes ne saurait nous créer l'obligation de les soustraire à l'action du progrès, d'empêcher leur régulation ou leur amélioration. Avec le concours des tribunaux indigènes eux-mêmes, il sera possible d'amener peu à peu une pacification rationnelle, une généralisation des usages compatibles avec la condition sociale des habitants et de rendre ces usages de plus en plus conformes non point à nos doctrines juridiques métropolitaines qui peuvent être opposées, mais aux principes fondamentaux du droit naturel, source première de toutes les législations».

Le droit naturel est susceptible de bien des interprétations. En l'occurrence, il signifie le droit du colonisateur, supposé le plus civilisé. En 1931, un autre

53 J.O., Débats parlementaires, séance de la Chambre des députés, 28 juillet 1885, p. 1066.

54 Cf. N.ROULAND, op.cit.(Anthropologie Juridique), 350sq. 
gouverneur, R.Delavignette, plus lucide, critique les tentatives de rédaction des coutumes ivoiriennes :

"Qu'est-ce qu'une coutume africaine où les peines sont européanisées? [...] La coutume a cessé en fait d'être animée spirituellement. En abolissant les ordalies dans l'administration de la preuve, en restreignant le serment sur les fétiches ou le Coran, en ne tenant pas compte des éléments surnaturels qui s'attachaient à la personne des juges (...) est-ce que nous n'avons pas vidé la coutume de sa substance? [...] Quand nous disons que nous jugeons selon la coutume, nous sous entendons que nous commençons par juger la coutume elle-même d'après le Code».

En Afrique noire française, le décret Jacquinot du 14 septembre 1951 fait du consentement des époux le fondement du mariage.

Et l'Amérique du Nord?

$\mathrm{Au}$ XIXe siècle, les Indiens peuvent devenir citoyens américains, mais à condition qu'ils manifestent clairement leur volonté d'abandonner leur culture autochtone $\mathrm{e}^{55}$. Chaque postulant devait abandonner son nom indien et on lui faisait tirer une dernière flèche pour bien marquer qu'il était fermement résolu à abandonner son mode de vie traditionnel. Les hommes devaient toucher une charrue, symbole de la vie laborieuse de fermier qui s'ouvrait devant eux. Quant aux femmes, elles recevaient un sac à ouvrage et un porte-monnaie, s'engageant par la même à économiser et à se dévouer à leur foyer, comme les femmes blanches.

Au milieu du XX siècle, les Etats Unis mettent en œuvre une Termination Policy qui visait à l'extinction des Nations Indiennes ${ }^{56}$.L'histoire en décidera autrement.

Quand on étudie l'histoire coloniale, on s'aperçoit donc que le colonisateur, ou, si l'on préfère, l'ethnie dominante, n'ont accepté les coutumes locales que sous bénéfice d'inventaire. Très souvent, la rédaction des coutumes n'a servi qu'à effectuer un tri entre celles-ci.

Et il faut bien remarquer qu'on ne peut pas considérer comme bonnes certaines coutumes : l'excision, le suicide des veuves, l'infanticide des petites filles (aujourd'hui, en Inde ou en Chine, grâce à l'échographie et aux diagnostics prénataux, l'infanticide des petites filles a atteint un niveau extrêmement important. Le déficit de naissances féminines se chiffre à plusieurs centaines de millions), les sacrifices humains, etc. C'est donc à la fois au législateur et aux populations locales d'entretenir un dialogue qui permette de faire le tri entre les bonnes et les mauvaises coutumes. Il n'y a que par la connaissance réciproque des institutions et des mentalités qu'on pourra y parvenir.

55 Cf. Norbert ROULAND, op.cit. ( Droit des minorités et des peuples autochtones), 373.

56 Cf. Norbert ROULAND (dir.), Droit des minorités et des peuples autochtones, Paris, PUF, 1996,PP. 374-375. 


\section{CONCLUSION}

Sur des sujets aussi vastes que le pluralisme juridique et le matriarcat, il est bien difficile de conclure. Le pluralisme juridique est certes une attitude de tolérance, mais il correspond également à des stratégies et reste plus difficile à définir que les systèmes unitaires, ce qui est logique. Quel qu'en soit le degré, la prise en compte des différences suppose toujours la mise en place d'institutions complexes. Mais préalablement elle nécessite la reconnaissance de l'altérité, ce qui n'est jamais une attitude spontanée. : la difficile coexistence entre les systèmes matristiques et patriarcaux le montre bien. On le voit notamment dans les relations affectives, où la recherche de l'identique marque en général le début des relations amoureuses. La maturité consiste au contraire dans l'acceptation des différences. Mais celle-ci ne peut être absolue. Il est des normes de comportement qui resteront toujours irréductibles : le terrorisme en est la preuve tragique. C'est aux philosophes, aux anthropologues et aux juristes de définir la bonne mesure de la complémentarité. 